\title{
Higgs-boson production in association with a dark photon in $\mathrm{e}^{+} \mathbf{e}^{-}$collisions
}

\author{
Sanjoy Biswas, ${ }^{a}$ Emidio Gabrielli, ${ }^{b, c, d}$ Matti Heikinheimo ${ }^{d}$ and Barbara Mele ${ }^{e}$ \\ ${ }^{a} K I A S$, \\ 85 Hoegi-ro, Dongdaemun-gu, Seoul, 130-722 Republic of Korea \\ ${ }^{b}$ Dipartimento di Fisica Teorica, Università di Trieste, \\ Strada Costiera 11, Trieste, I-34151 Italy \\ ${ }^{c}$ INFN, Sezione di Trieste, \\ Via Valerio 2, Trieste, I-34127 Italy \\ ${ }^{d} \mathrm{NICPB}$, \\ Ravala 10, Tallinn, 10143 Estonia \\ eINFN, Sezione di Roma, c/o Dipartimento di Fisica, Università di Roma "La Sapienza", \\ P.le Aldo Moro 2, Rome, I-00185 Italy \\ E-mail: sanjoy@kias.re.kr, emidio.gabrielli@cern.ch, \\ matti.heikinheimo@kbfi.ee, barbara.mele@roma1.infn.it
}

ABSTRACT: We study the production of a Higgs boson recoiling from a massless invisible system in $e^{+} e^{-}$collisions. This is a quite distinctive signature that can arise when the Higgs boson is produced in association with a massless dark photon, which can happen in BSM scenarios foreseeing an extra unbroken $\mathrm{U}(1)$ gauge group. Dark photons can indeed acquire effective couplings to the Higgs boson as occurs in models recently proposed to generate exponentially-spread Yukawa couplings. We analyze the signal and corresponding backgrounds for $H \rightarrow b \bar{b}$, and estimate ILC and FCC-ee sensitivities in a model-independent way.

Keywords: Higgs Physics, Beyond Standard Model

ARXiv EPrint: 1503.05836 


\section{Contents}

1 Introduction 1

2 Effective dark-photon couplings to the Higgs boson 4

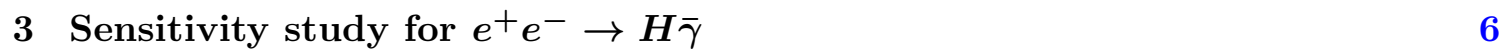

4 A model of Flavor with dark photons $\quad 11$

$\begin{array}{lll}4.1 \text { The Lagragian } & 12\end{array}$

$\begin{array}{lll}4.2 & \operatorname{BR}(H \rightarrow \gamma \bar{\gamma}) \text { predictions } & 14\end{array}$

$\begin{array}{lll}5 & \text { Conclusions } & 19\end{array}$

$\begin{array}{ll}\text { A Coupling strength in the dark sector } & 20\end{array}$

\section{Introduction}

The LHC discovery of the Higgs-boson resonance at $125 \mathrm{GeV}[1,2]$ has definitely strengthened our confidence in the Higgs mechanism as the origin of the electroweak (EW) symmetry breaking (EWSB) and fermion mass generation [3-6]. All present data are well consistent with the Standard Model (SM) expectations for the Higgs boson [7, 8], although there is still room for potential New Physics (NP) effects.

At the same time, the absence of any NP signal at the LHC Run I is causing considerable concern about the applicability of the naturalness criteria. The latter would require new phenomena at the TeV scale to stabilize the SM Higgs scalar potential against potentially large radiative corrections coming from NP energy thresholds. The SM is also facing the Flavor problem, which is related to the unexplained huge hierarchy in the fermion mass spectrum or, analogously, in the Higgs Yukawa couplings.

On another front, there is increasing evidence from astrophysical and cosmological observations of the existence of Dark Matter (DM) in the Universe $[9,10]$, which is not predicted in the SM. Independently of naturalness criteria, there might then be NP above the EW scale that explains DM and, in some cases, could be tested at the LHC.

A common origin for DM and Flavor is also conceivable. One can postulate the existence of a hidden (dark) sector, where all these issues are addressed, which is composed of new fields that are SM singlets. The Higgs boson can then act as a portal to the dark sector [11]. The Flavor and EWSB structures are indeed restricted to the Higgs couplings and mass, and are not related to other SM couplings. On the other hand, NP could well affect the Higgs-boson characteristics by smaller amounts than the present LHC sensitivity in Higgs-boson data. 

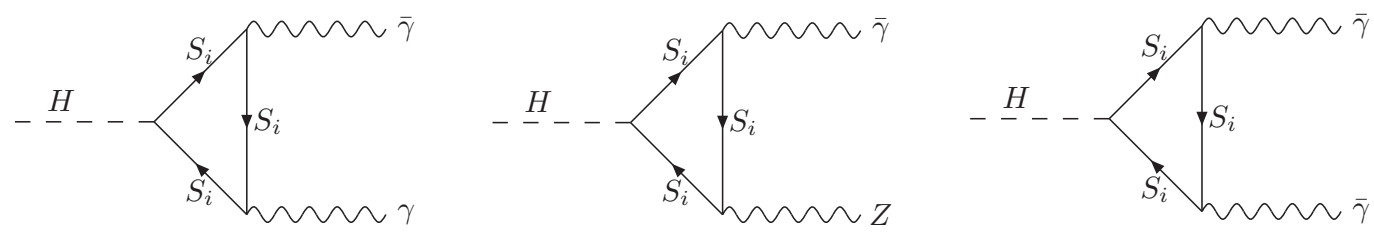

Figure 1. Higgs decays $H \rightarrow \gamma \bar{\gamma}, Z \bar{\gamma}, \bar{\gamma} \bar{\gamma}$, via mediator loops.

In this connection, in [12] a new paradigm has been proposed to generate exponentiallyspread Yukawa couplings from gauge quantum numbers in the dark sector. In this class of models, the Flavor and chiral symmetry breaking (ChSB) take place in a dark sector, and are transmitted by radiative corrections to the observable sector through Higgs-portal type of interactions. The Yukawa couplings arise radiatively as effective couplings at low energy. The hidden sector consists of (stable) massive dark fermions (that are SM singlets and potential DM candidates), and a massless dark photon, the gauge boson of an unbroken $\mathrm{U}(1)_{F}$ gauge group in the dark sector. Chiral symmetry is spontaneously broken, and dark fermions obtain non-vanishing (Flavor-dependent) masses via a non-perturbative mechanism involving $\mathrm{U}(1)_{F}$ gauge interactions. The resulting chiral-symmetry and Flavor breaking in the dark sector is then transferred to the Yukawa-coupling sector at one-loop via scalar messenger fields that are charged under both $\mathrm{SM}$ and $\mathrm{U}(1)_{F}$ gauge interactions. A similar framework has also been explored in [13-15], although no unbroken $\mathrm{U}(1)$ gauge sector is introduced in that case.

The new unbroken U(1) gauge group (and the corresponding massless dark photon) is a crucial dynamical component of the model in [12], but is also a common feature of various theories of new physics, including models with gauge-symmetry breaking of compact gauge groups, string-theory motivated phenomenological models, and models of interacting dark matter [16-28]. It is indeed conceivable that a hidden sector contains an extra long-range force. Remarkably, being massless, an on-shell dark photon can be fully decoupled from the SM quark and lepton sector at any order in perturbation theory [16]. This is not true for a massive dark-photon, due to a potential tree-level mixing with the photon field. Most of present astrophysical and accelerator constraints [29-34] apply to massive darkphoton couplings, and can be evaded in a massless dark-photon scenario. This allows for potentially large dark-photon couplings to the dark sector, that might also lead to observable new signatures at colliders [12].

The Higgs boson can interact with dark photons radiatively. In the framework proposed in [12], this occurs at one loop by the exchange of scalar messenger fields (figure 1).

As a consequence, the Higgs boson can act as a portal toward the dark sector, giving rise to new Higgs-boson decays such as [35]

$$
H \rightarrow \gamma \bar{\gamma}, Z \bar{\gamma}, \bar{\gamma} \bar{\gamma}
$$

where the symbols $\gamma$ and $\bar{\gamma}$ stand for the usual QED photon and dark photon, respectively, and $Z$ is the neutral vector boson. The corresponding decay rates can in principle be large, even for very heavy messenger fields. As in the $H \rightarrow \gamma \gamma, Z \gamma, g g$ decays in the SM, the 


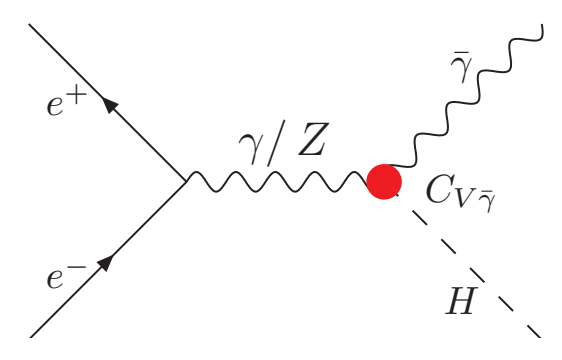

Figure 2. Diagrams for $e^{+} e^{-} \rightarrow H \bar{\gamma}$.

non-decoupling Higgs properties guarantee non-vanishing decay widths even in the largemass limit for particles exchanged in the loop, provided the virtual (messenger) fields carry the same $\mathrm{SU}(2)_{L}$ quantum numbers of quarks and leptons.

Being fully decoupled at tree level from the SM sector, a single massless dark photon will give rise in the Higgs final state to same amount of missing energy and missing momentum, while the two dark-photon channel will contribute to the invisible Higgs rate. Extra contributions to the widths of the SM channels $H \rightarrow \gamma \gamma, Z \gamma, g g$ are also expected in general.

The $H \rightarrow \gamma \bar{\gamma}$ decay gives rise to a new spectacular signature at the LHC in $\gamma+E / T$ final states, with a photon plus missing transverse energy E/T resonating at the Higgs mass. In [35], a parton-level study shows that the LHC Run-1 data set could be sensitive to $\mathrm{BR}(H \rightarrow \gamma \bar{\gamma})$ values as low as $0.5 \%$, while a minimal-model prediction for $\mathrm{BR}(H \rightarrow \gamma \bar{\gamma})$ can be as large as $5 \%$.

The aim of the present study is to analyze the phenomenological implications of the Higgs effective couplings to dark photons at future $e^{+} e^{-}$colliders [36-39]. Apart from the new signatures corresponding to the Higgs-boson exotic decays in eq. (1.1) (that we do not address here), effective $H \bar{\gamma} \gamma$ and $H \bar{\gamma} Z$ interactions involving dark photons will give rise to final states with a Higgs boson and a dark photon,

$$
e^{+} e^{-} \rightarrow H \bar{\gamma}
$$

via $s$-channel exchange of either a photon or a $Z$ vector boson (figure 2). This channel, although kinematically similar to the SM one-loop channel $e^{+} e^{-} \rightarrow H \gamma$ [40-43], gives rise to a completely new signature, since the final massless $\bar{\gamma}$ goes undetected.

We will focus on the $b \bar{b} \bar{\gamma}$ final state corresponding to the main Higgs decay channel $H \rightarrow b \bar{b}$, although even more rare Higgs decays will be of relevance in the clean $e^{+} e^{-}$environment $[44,45]$. The $e^{+} e^{-} \rightarrow H \bar{\gamma}$ final state will then be characterized by an unbalanced $b \bar{b}$ system resonating at the Higgs mass $m_{\mathrm{H}}$, the dark photon $\bar{\gamma}$ giving rise to "monochromatic" missing energy $\not E$ and momentum $\not p$ (for fixed initial c.m. collision energy $\sqrt{s}$ ). Contrary to what occurs in the main irreducible SM $b \bar{b} \nu \bar{\nu}$ background, at parton level the invariant mass of the invisible system $M_{\text {miss }}=\left(E^{2}-\not p^{2}\right)^{1 / 2}$ vanishes. This feature will provide a crucial handle for background suppression.

Since the messenger fields are expected to be quite heavy with respect to the characteristic energy of the $e^{+} e^{-} \rightarrow H \bar{\gamma}$ process, the $H \gamma \bar{\gamma}$ and $H Z \bar{\gamma}$ vertices can be considered 


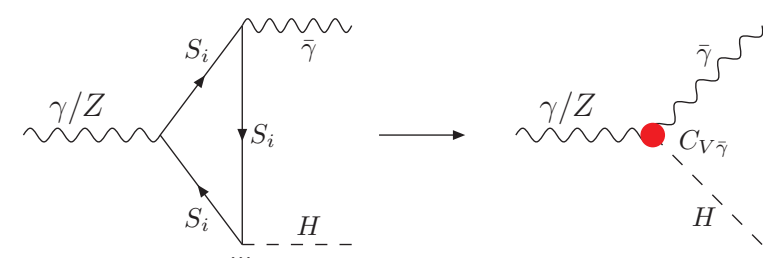

Figure 3. Effective coupling approximation for the vertices $H \gamma \bar{\gamma}, H Z \bar{\gamma}$.

as effective interactions, and described by a model-independent parametrization [35]. The ratio of the $H \bar{\gamma} Z$ and $H \bar{\gamma} \gamma$ couplings will in general depend on the spin and the SM gauge-group representation of the new particles running in the loop. For simplicity, we will focus here on scenarios where the $H \bar{\gamma} Z$ vertex is induced by scalar messenger fields in the $\mathrm{SU}(2)_{L} \times \mathrm{SU}(3)_{c}$ fundamental representation [12], which gives a definite prediction for the $H \bar{\gamma} Z$ and $H \bar{\gamma} \gamma$ coupling ratio.

The paper is organized as follows. In section 2, we provide a model-independent parametrization of the effective couplings controlling the Higgs exotic decays $H \rightarrow \gamma \bar{\gamma}$, $Z \bar{\gamma}, \bar{\gamma} \bar{\gamma}$, and the SM-like decays $H \rightarrow \gamma \gamma, Z \gamma$, and express the relevant Higgs BR's in terms of the model-independent coefficients. In section 3 , we study the sensitivity of future $e^{+} e^{-}$colliders to the $e^{+} e^{-} \rightarrow H \bar{\gamma}$ associated production by analyzing the signal and corresponding backgrounds. In section 4 , we discuss the NP model in [12] that aims to solve the Flavor hierarchy problem. We also present the corresponding predictions for the Higgs-dark-photon effective couplings, and for the Higgs branching ratios (BR's) relative to the decays $H \rightarrow \gamma \bar{\gamma}$, and $H \rightarrow Z \bar{\gamma}$. Finally, our conclusions are discussed in section 5 . In the appendix, we describe some $\mathrm{U}(1)_{F}$ coupling properties of the model in [12], that are needed to discuss its phenomenological consequences.

\section{Effective dark-photon couplings to the Higgs boson}

We now introduce the dark-photon effective couplings to the Higgs boson that enter the $e^{+} e^{-} \rightarrow H \bar{\gamma}$ cross section. In general, Higgs-dark-photon effective couplings can arise at one loop due to the exchange of messenger fields that are charged under both the SM and the $\mathrm{U}(1)_{F}$ gauge groups (figure 3 ). In case the messenger masses are much larger than both $m_{\mathrm{H}}$ and $\sqrt{s}$, one can use the effective theory approximation. The corresponding effective Lagrangian $\mathcal{L}_{\text {eff }}^{\text {Higgs }}$ can be split as

$$
\mathcal{L}_{\text {eff }}^{\text {Higgs }}=\mathcal{L}_{\mathrm{DP}_{\mathrm{H}}}+\mathcal{L}_{\mathrm{SM}_{\mathrm{H}}},
$$

where $\mathcal{L}_{\mathrm{DP}_{\mathrm{H}}}$ contains the dark-photon effective interactions with the Higgs boson, while $\mathcal{L}_{\mathrm{SM}_{\mathrm{H}}}$ presents the extra (that is messenger-induced) contributions to the SM Higgs effective interactions with two photons, one photon and a $Z$, and two gluons.

By retaining only the relevant low-energy operators, $\mathcal{L}_{\mathrm{DP}_{\mathrm{H}}}$ can be expressed in terms of dimensionless (real) coefficients $C_{i j}$ (with $i, j=\bar{\gamma}, \gamma, Z, g$ ) as

$$
\mathcal{L}_{\mathrm{DP}_{\mathrm{H}}}=\frac{\alpha}{\pi}\left(\frac{C_{\gamma \bar{\gamma}}}{v} \gamma^{\mu \nu} \bar{\gamma}_{\mu \nu} H+\frac{C_{Z \bar{\gamma}}}{v} Z^{\mu \nu} \bar{\gamma}_{\mu \nu} H+\frac{C_{\bar{\gamma} \bar{\gamma}}}{v} \bar{\gamma}^{\mu \nu} \bar{\gamma}_{\mu \nu} H\right)
$$


where $\alpha$ is the SM fine structure constant, and $\gamma_{\mu \nu}, Z_{\mu \nu}, \bar{\gamma}_{\mu \nu}$ are the field strentghs of photon, $Z$ boson, and dark photon, respectively $\left(\gamma_{\mu \nu} \equiv \partial_{\mu} A_{\nu}-\partial_{\nu} A_{\mu}\right.$ for the photon field $\left.A_{\mu}\right)$. Then, $\mathcal{L}_{\mathrm{SM}_{\mathrm{H}}}$ can be written as

$$
\mathcal{L}_{\mathrm{SM}_{\mathrm{H}}}=\frac{\alpha}{\pi}\left(\frac{C_{\gamma \gamma}}{v} \gamma^{\mu \nu} \gamma_{\mu \nu} H+\frac{C_{Z \gamma}}{v} Z^{\mu \nu} \gamma_{\mu \nu} H\right)+\frac{\alpha_{\mathrm{S}}}{\pi} \frac{C_{g g}}{v} G^{a \mu \nu} G_{\mu \nu}^{a} H
$$

where $\alpha_{\mathrm{S}}$ is the SM strong coupling constant, $G^{a \mu \nu}$ stands for the gluon field strength, and a sum over the color index $a$ is understood.

As usual, the $C_{i j}$ coefficients in eqs. (2.2)-(2.3) can be computed in the complete theory by evaluating one-loop amplitudes for relevant physical processes, and by matching them with the corresponding results obtained at tree level via the effective Lagrangian in eq. (2.1). In particular, in order to express the coefficients $C_{\gamma \bar{\gamma}}, C_{Z \bar{\gamma}}, C_{\bar{\gamma} \bar{\gamma}}$ in eq. (2.2) in terms of the fundamental parameters of the model, one can match the tree-level widths, based on the parametrization in eq. (2.2), for the Higgs decays $H \rightarrow \gamma \bar{\gamma}, H \rightarrow Z \bar{\gamma}, H \rightarrow \bar{\gamma} \bar{\gamma}$, respectively, with the corresponding one-loop results computed in the full model (as sketched in figure 3 ). This will be discussed in section 4, after introducing a particular NP framework.

On the other hand, one can perform a phenomenological study of the $e^{+} e^{-} \rightarrow H \bar{\gamma}$ process just on the basis of the model-independent parametrization in eq. (2.2), which we will do in the next section.

Before proceeding, we connect the basic $C_{i j}$ coefficients in eq. (2.2) to the corresponding $H \rightarrow i j$ decay widths. The $H \rightarrow \gamma \bar{\gamma}$ width has been computed in [35], and, taking into account the parametrization in eq. (2.2), one has

$$
\Gamma(H \rightarrow \gamma \bar{\gamma})=\frac{m_{\mathrm{H}}^{3}}{64 \pi \Lambda_{\gamma \bar{\gamma}}^{2}}, \quad 1 / \Lambda_{\gamma \bar{\gamma}}=C_{\gamma \bar{\gamma}} /(\pi v)
$$

Analogous results can be obtained for the $H \rightarrow \bar{\gamma} \bar{\gamma}, H \rightarrow Z \bar{\gamma}, H \rightarrow \gamma \gamma$ widths replacing $1 / \Lambda_{\gamma \bar{\gamma}}$ by $1 / \Lambda_{\bar{\gamma} \bar{\gamma}}=C_{\bar{\gamma} \bar{\gamma}} /(\pi v), 1 / \Lambda_{Z \bar{\gamma}}=C_{Z \bar{\gamma}} /(\pi v), 1 / \Lambda_{\gamma \gamma}=\left(C_{\gamma \gamma}^{\mathrm{SM}}+C_{\gamma \gamma}\right) /(\pi v)$, respectively.

Figure 4 shows the branching ratios for $H \rightarrow \gamma \bar{\gamma}$ and $H \rightarrow Z \bar{\gamma}$, normalized to the SM $\operatorname{BR}(H \rightarrow \gamma \gamma)$ and $\operatorname{BR}(H \rightarrow Z \gamma)$, respectively, versus the corresponding $C_{i j}$ coefficients. The $C_{i j}$ ranges shown in the plot include values well allowed by the model described in section 4. One can then get for the Higgs decays into a dark photon an enhancement factor $\mathcal{O}(10)$ with respect to the SM Higgs decays where the dark photon is replaced by a photon. This makes the corresponding phenomenology quite relevant for both LHC and future-collider studies.

It is also useful to express the BR's for $H \rightarrow \gamma \bar{\gamma}, \bar{\gamma} \bar{\gamma}, \gamma \gamma$ as a function of the relative exotic contribution $r_{i j}$ to the $H \rightarrow i j$ decay width, as the ratio

$$
r_{i j} \equiv \frac{\Gamma_{i j}^{\mathrm{m}}}{\Gamma_{\gamma \gamma}^{\mathrm{SM}}}
$$

with $\Gamma_{i j}^{\mathrm{m}}$ generically indicating the pure messenger contribution to $H \rightarrow i j$, with $i, j=\gamma, \bar{\gamma}$. 


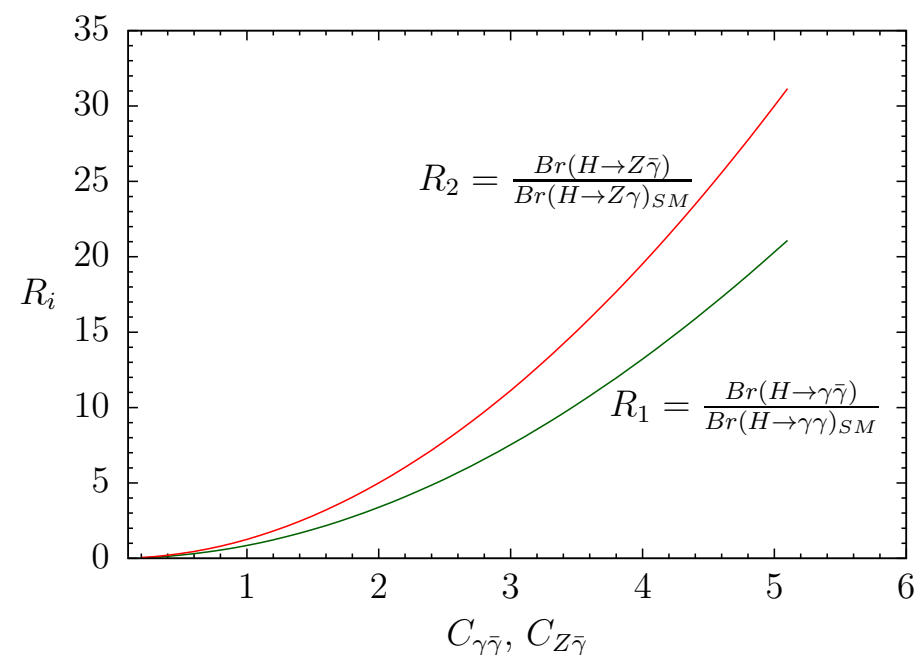

Figure 4. BR's for $H \rightarrow \gamma \bar{\gamma}$ and $H \rightarrow Z \bar{\gamma}$, normalized to the SM values for $\mathrm{BR}(H \rightarrow \gamma \gamma)$ and $\operatorname{BR}(H \rightarrow Z \gamma)$, respectively, versus $C_{\gamma \bar{\gamma}}$ and $C_{Z \bar{\gamma}}$.

Then, one obtain the following model-independent parametrization of the $H \rightarrow \gamma \bar{\gamma}$, $\bar{\gamma} \bar{\gamma}, \gamma \gamma$ BR's as functions of $r_{i j}[35]$

$$
\begin{aligned}
\mathrm{BR}_{\gamma \bar{\gamma}} & =\mathrm{BR}_{\gamma \gamma}^{\mathrm{SM}} \frac{r_{\gamma \bar{\gamma}}}{1+r_{\bar{\gamma} \bar{\gamma}} \mathrm{BR}_{\gamma \gamma}^{\mathrm{SM}}}, \\
\mathrm{BR}_{\bar{\gamma} \bar{\gamma}} & =\mathrm{BR}_{\gamma \gamma}^{\mathrm{SM}} \frac{r_{\bar{\gamma} \bar{\gamma}}}{1+r_{\bar{\gamma} \bar{\gamma}} \mathrm{BR}_{\gamma \gamma}^{\mathrm{SM}}}, \\
\mathrm{BR}_{\gamma \gamma} & =\mathrm{BR}_{\gamma \gamma}^{\mathrm{SM}} \frac{\left(1+\chi \sqrt{r_{\gamma \gamma}}\right)^{2}}{1+r_{\bar{\gamma} \bar{\gamma}} \mathrm{BR}_{\gamma \gamma}^{\mathrm{SM}}},
\end{aligned}
$$

where $\chi= \pm 1$ parametrizes the relative sign of the $\mathrm{SM}$ and exotic amplitudes, and $\mathrm{BR}_{i j}$ stands for $\operatorname{BR}(H \rightarrow i j)$.

Analogously, the relative deviation for the $H \rightarrow g g$ decay width will be defined as

$$
r_{g g} \equiv \frac{\Gamma_{g g}^{\mathrm{m}}}{\Gamma_{g g}^{\mathrm{SM}}} .
$$

\section{Sensitivity study for $e^{+} e^{-} \rightarrow H \bar{\gamma}$}

We focus now on the $\bar{\gamma}$ production in association with a Higgs boson in $e^{+} e^{-}$collisions. The $e^{+} e^{-} \rightarrow H \bar{\gamma}$ total cross section versus $\sqrt{s}$ is shown in figure 5 for three different coupling assumptions: $C_{\gamma \bar{\gamma}}=1, C_{Z \bar{\gamma}}=0$ (blue line); $C_{\gamma \bar{\gamma}}=0, C_{Z \bar{\gamma}}=1$ (green line); $C_{\gamma \bar{\gamma}}=1, C_{Z \bar{\gamma}}=0.79 C_{\gamma \bar{\gamma}}$ (red line). The coupling ratio $C_{Z \bar{\gamma}} / C_{\gamma \bar{\gamma}} \simeq 0.79$ is typical for scenarios where the $H \bar{\gamma} Z$ vertex is induced by scalar messenger fields in the $\mathrm{SU}(2)_{L} \times \mathrm{SU}(3)_{c}$ fundamental representation (see section 4 ). The corresponding cross sections at $\sqrt{s} \simeq 1 \mathrm{TeV}$ (relevant for linear colliders at larger collision energy) are $43 \mathrm{ab}, 15 \mathrm{ab}, 55 \mathrm{ab}$, respectively. Cross sections can be easily extrapolated to coupling set-up obtained just by globally rescaling these set of couplings. 


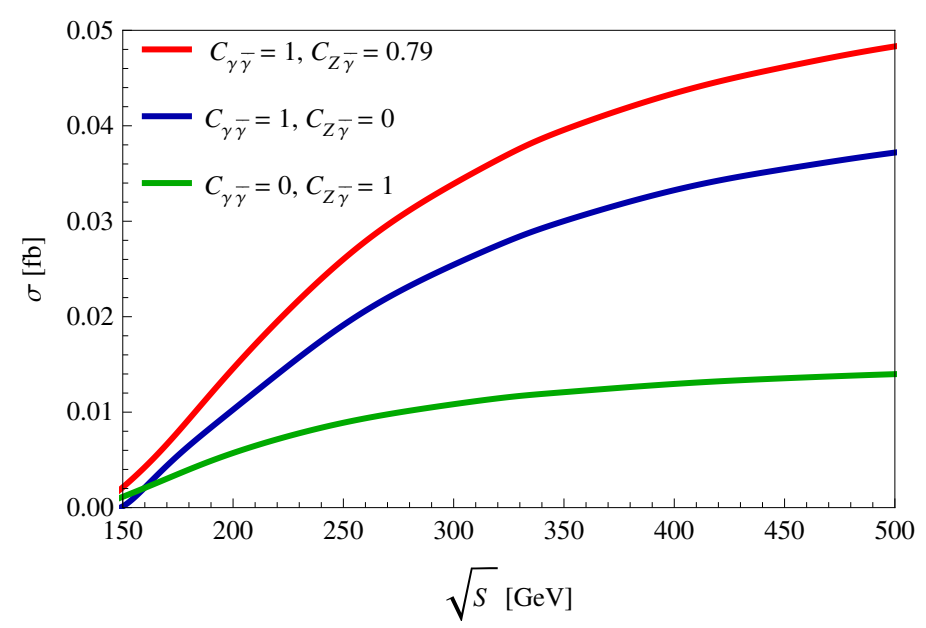

Figure 5. Total $e^{+} e^{-} \rightarrow H \bar{\gamma}$ cross section as a function of the c.m. collision energy, for different sets of effective couplings.

The $e^{+} e^{-} \rightarrow H \bar{\gamma}$ cross sections grow with c.m. energy thanks to the nature of the dimension-five operators in the effective Lagrangian in eq. (2.2). Hence, at constant integrated luminosity, higher-energy colliders will have a higher potential, since the dominant background is expected to scale down with energy as $1 / s$. On the other hand, lower $\sqrt{s}$ may allow larger integrated luminosity, as is the case of the $e^{+} e^{-}$Future Circular Collider (FCC-ee) (also called TLEP) [38, 39], where an integrated luminosity of $10 \mathrm{ab}^{-1}$ is expected at $\sqrt{s}=240 \mathrm{GeV}$. At linear colliders, either ILC [36] or CLIC [37], one typically foresees integrated luminosities of a few hundreds $\mathrm{fb}^{-1}$ in the initial energy of $\sqrt{s} \sim 250 \mathrm{GeV}$ or $350 \mathrm{GeV}$, and a few $\mathrm{ab}^{-1}$ at the larger- $\sqrt{s}$ stages [46]. Here, we assume the minimal energy setup of $\sqrt{s}=240 \mathrm{GeV}$ that is relevant for Higgs-boson studies, and study the sensitivity to $e^{+} e^{-} \rightarrow H \bar{\gamma}$ production versus integrated luminosities foreseen at different machines.

Using the effective Lagrangian eq. (2.2) implemented by FeynRules [47], we have generated $e^{+} e^{-} \rightarrow H \bar{\gamma} \rightarrow b \bar{b} \bar{\gamma}$ events with MadGraph5_aMC@NLO [48], and passed these events to PYTHIA to account for parton showering, and hadronization. We checked that the inclusion of effects from initial state radiation, that tends to degrade the c.m. energy in a circular $e^{+} e^{-}$colliders, would moderately affect the results of the present analysis. We neither include beamstrahlung effects that can be of some relevance at linear colliders. We account for finite detector resolution by applying the jet-energy smearing $\sigma(E) / E=30 \% / \sqrt{E}$, which is typical for ILC-kind of detectors [49] .

The dark photon escapes the experimental apparatus undetected, and the final signal consists of two $b$ quarks and large missing energy $E$ and momentum $\not p$. In our simulation we reconstruct the missing momentum from the vector sum of all visible final-state particle momenta, after applying PYTHIA. In a lepton collider a $H \rightarrow b \bar{b}$ final state is not swamped by large QCD backgrounds, as occurs in hadronic collisions. Therefore, $b \bar{b}$ final states are the best channel to search for $H \bar{\gamma}$ production, thanks to the $H \rightarrow b \bar{b}$ large rate. After showering and hadronization, we reconstruct jets (and $b$-jets) according to the basic PYTHIA jet-cone algorithm, assuming a quite large cone aperture $R_{j}=1.5$, which 
optimizes mass reconstruction [50]. The basic event selection is given by

$$
p_{T}^{b}>20 \mathrm{GeV}, \quad\left|\eta_{b}\right|<2.5, \quad \Delta R(b b)>0.4, \quad E>40 \mathrm{GeV},
$$

where $\Delta R(b b)=\sqrt{\Delta \eta^{2}+\Delta \phi^{2}}$ is the angular distance between two $b$-tagged jets. We assume a $b$-tagging efficiency of $80 \%$, and a corresponding fake $b$-jet rejection factor of 100 for light jets.

The main SM background for the $b \bar{b}+\not E$ final state is given by the $\nu \bar{\nu} b \bar{b}$ production. This includes the on-shell processes $Z Z \rightarrow \nu \bar{\nu} b \bar{b}, Z H \rightarrow \nu \bar{\nu} b \bar{b}$, which give an almost monochromatic $b \bar{b}$-pair system (similarly to the signal), and the vector boson fusion channel $H \nu \bar{\nu}$. A subdominant contribution comes from $\nu \bar{\nu} q \bar{q}$ (mostly from on-shell $Z$ pairs), where both light jets are mis-tagged as $b$ jets.

There are two kinematical variables that turn out to be particularly efficient in separating the signal from the background. First, we introduce the variable $M_{j j}$ as the invariant mass of the two jets with largest $p_{T}$. This is directly connected to the $b$-pair invariant mass, and can be used to pinpoint events with $b$-quarks coming from Higgs decays, out of the smaller- $M_{j j}$ events arising from $Z \rightarrow b \bar{b}$. There is anyway part of the $\nu \bar{\nu} b \bar{b}$ background that goes through the $Z H$ production resonating at $M_{j j} \sim m_{\mathrm{H}}$, just as in the signal case. This is well illustrated by figure 6 , where the normalized invariant-mass distributions of the $b \bar{b}$ system are compared for signal and backgrounds. Second, we introduce the missing-mass variable $M_{\text {miss }}$, defined as

$$
M_{\text {miss }}=\sqrt{k^{2}-p^{2}},
$$

where $E=\sqrt{s}-\sum \mathrm{E}_{\text {visible }}$ and $\not \mathbf{p}=-\sum \mathbf{p}_{\text {visible }}$ are the final-state missing energy and missing three-momentum vector, respectively (the sum over visible objects here includes both jets and lower-energy particles escaping jet reconstruction). The $M_{\text {miss }}$ variable is expected to approximately vanish in the partonic description of $e^{+} e^{-} \rightarrow H \bar{\gamma}$, corresponding to the massless invisible dark photon. A cut on $M_{\text {miss }}$ then proves to be remarkably efficient in further separating the signal from the main background, where $M_{\text {miss }}$ mostly matches an invisible $Z$-boson decaying into neutrinos.

The $M_{\text {miss }}$ spectrum of the signal and background processes are compared in figure 7 , after applying PYTHIA showering, jet reconstruction and jet-energy resolution effects on top of parton-level simulation (right panel). The parton-level spectrum, shown in the left panel of the same figure, shows a distinct peak at $M_{\text {miss }} \simeq 0$ for the signal, and at $M_{\text {miss }} \sim M_{Z}$ for the background processes. No energy-resolution effect has been applied in the latter case, and the smearing of the peaks is just due to the presence of neutrinos from $b$ decays, and to the possible off-shellness of the $\nu \bar{\nu}$ system in the background. Applying the parton showering, jet reconstruction and energy-resolution effects (as in the right panel of the figure) degrades the $M_{\text {miss }}$ spectrum of the signal quite a lot, shifting the peak away from zero and smearing it. Hence, an optimal detector resolution would be particularly crucial in this analysis.

On the basis of the $M_{j j}$ and $M_{\text {miss }}$ distributions in figures 6 and 7, we set a suitable event selection. We require the invariant mass $M_{j j}$ to be within $10 \%$ of the $M_{j j}$ peak value of the simulated signal events, and then impose the missing mass to be below $40 \mathrm{GeV}$. The 


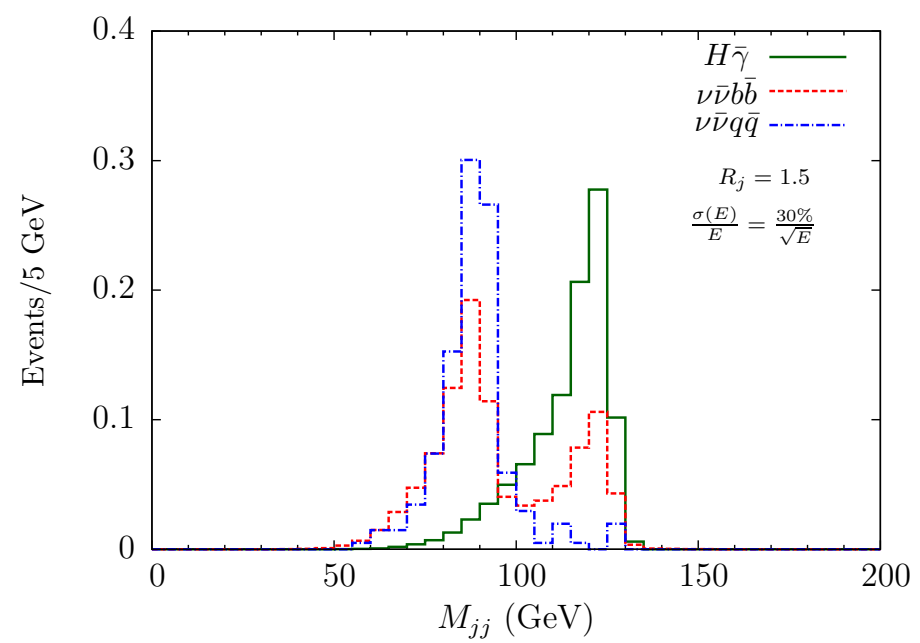

Figure 6. Invariant-mass $M_{j j}$ distributions for the two jets with largest $p_{T}$ for the the signal (solid line) and the two backgrounds $\nu \bar{\nu} b \bar{b}$ (dashed line) and $\nu \bar{\nu} q \bar{q}$ (dot-dashed line) after PYTHIA showering, hadronization, and jet-energy resolution effect. All distributions are normalized to 1.
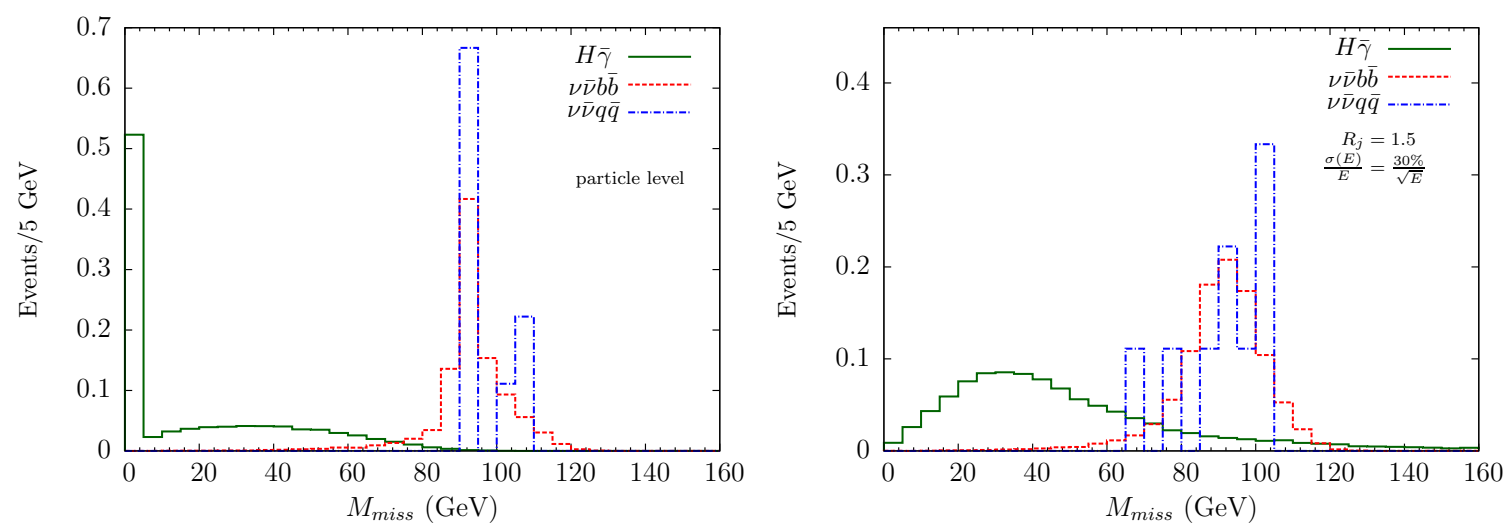

Figure 7. $M_{\text {miss }}$ distributions for the the signal (solid line) and the backgrounds $\nu \bar{\nu} b \bar{b}$ (dashed line) and $\nu \bar{\nu} q \bar{q}$ (dot-dashed line) after parton level simulation (left) and after PYTHIA showering, hadronization and jet energy resolution effect (right). All distributions are normalized to 1.

latter cuts make the $\nu \bar{\nu} q \bar{q}$ background negligible. The $\nu \bar{\nu} b \bar{b}$ background can still be slightly reduced after these cuts by making a further cut on the missing energy $E$. The $E$ spectrum is shown in figure 8 for the signal and background events satisfying the previous $M_{j j}$ and $M_{\text {miss }}$ cuts. Both the signal and background distributions peak at around the same value, with the background moderately shifted to larger $E$ values. Thus we require the missing energy to be below $100 \mathrm{GeV}$. Including the initial event selection criteria, we altogether impose that the missing energy satisfies the condition $40 \mathrm{GeV}<E<100 \mathrm{GeV}$.

Table 1 shows the cross sections and the acceptances for the signal and the $\nu \bar{\nu} b \bar{b}$ background after applying the cut-flow just described, for $\sqrt{s}=240 \mathrm{GeV}$. The signal acceptance is practically insensitive to to a change in the relative contribution of the $C_{\gamma \bar{\gamma}}$ and $C_{Z \bar{\gamma}}$ couplings. The corresponding acceptance for the $\nu \bar{\nu} q \bar{q}$ background is negligible.

On the basis of the table 1 acceptances, we can work out the expected sensitivity to the signal for given values of the $C_{\gamma \bar{\gamma}}, C_{Z \bar{\gamma}}$ couplings. As usual, we define the signal 


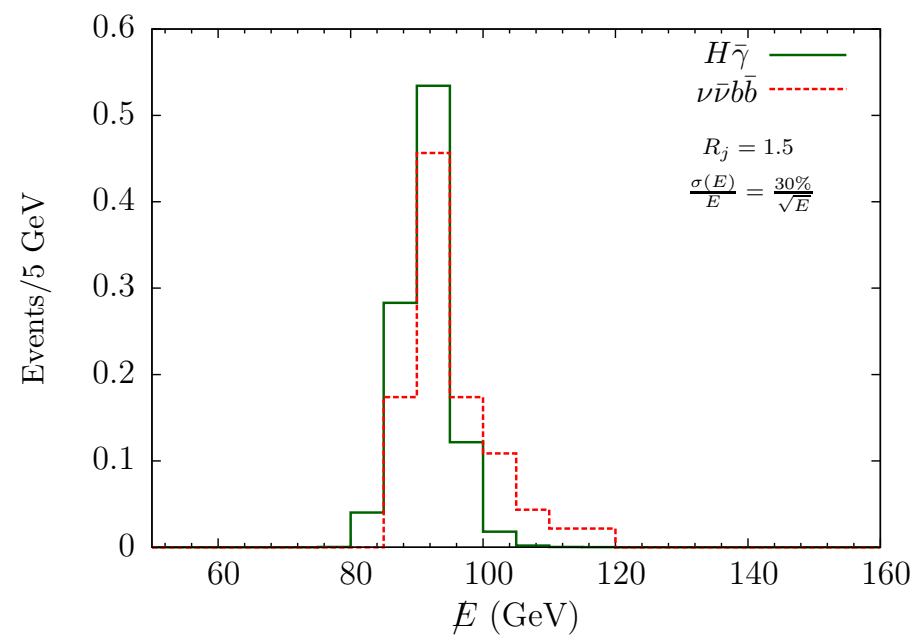

Figure 8. $\not E$ distributions for the the signal (solid line) and the background $\nu \bar{\nu} b \bar{b}$ (dashed line) after PYTHIA showering, hadronization and jet-energy resolution effect, and after applying the $M_{j j}$ and $M_{\text {miss }}$ cuts described in the text. All distributions are normalized to 1.

\begin{tabular}{|ll|c|c|}
\hline \multicolumn{2}{|l|}{ Process } & Cross section (fb) & Acceptance after cuts (\%) \\
\hline$H \bar{\gamma} \quad\left(C_{Z \bar{\gamma}}=0\right)$ & $10.1 \times 10^{-3} C_{\gamma \bar{\gamma}}^{2}$ & 17.3 \\
\hline$H \bar{\gamma} \quad\left(C_{\gamma \bar{\gamma}}=0\right)$ & $4.8 \times 10^{-3} C_{Z \bar{\gamma}}^{2}$ & 17.3 \\
\hline$H \bar{\gamma} \quad\left(C_{Z \bar{\gamma}}=0.79 C_{\gamma \bar{\gamma}}\right)$ & $13.8 \times 10^{-3} C_{\gamma \bar{\gamma}}^{2}$ & 17.3 \\
\hline SM $\nu \bar{\nu} b \bar{b}$ & 115. & 0.08 \\
\hline
\end{tabular}

Table 1. Cross sections (in $\mathrm{fb}$ ) and corresponding acceptances after kinematical cuts on signal and SM background at $\sqrt{s}=240 \mathrm{GeV}$. Applied cuts include the initial event selection in eq. (3.1), $M_{j j}$ to be within $10 \%$ of the $M_{j j}$ peak value of signal events, $M_{\text {miss }}<40 \mathrm{GeV}$, and $E<<100 \mathrm{GeV}$. Cross sections include $\operatorname{BR}(H \rightarrow b \bar{b}) \simeq 0.58$.

significance as $S / \sqrt{S+B}$, being $S$ and $B$ the event numbers for signal and background, respectively. Figure 9 shows the integrated luminosity needed to make a $5 \sigma$ observation of the $H \bar{\gamma}$ production in $e^{+} e^{-}$collisions at $\sqrt{s}=240 \mathrm{GeV}$, for any given value of the $C_{\gamma \bar{\gamma}}, C_{Z \bar{\gamma}}$ couplings (shown on the $y$-axis) when $C_{\gamma \bar{\gamma}}=0$ (green line), $C_{Z \bar{\gamma}}=0$ (blue line) and $C_{Z \bar{\gamma}}=0.79 C_{\gamma \bar{\gamma}}$ (red line).

For an integrated luminosity of $10 \mathrm{ab}^{-1}$ at $\sqrt{s}=240 \mathrm{GeV}$ (a typical value for FCCee), figure 10 shows the signal significance as a function of the couplings, with the same color convention as in figure 9 . The horizontal gray lines show the $5 \sigma$-discovery bound on couplings, and the $2 \sigma$ level approximating the $95 \%$ confidence-level exclusion.

Then, at $95 \%$ C.L., one can exclude the ranges $C_{\gamma \bar{\gamma}}>1.9$ (for $C_{Z \bar{\gamma}}=0$ ), $C_{Z \bar{\gamma}}>2.7$ (for $C_{\gamma \bar{\gamma}}=0$ ), and $C_{\gamma \bar{\gamma}}>1.6$ (for $C_{Z \bar{\gamma}}=0.79 C_{\gamma \bar{\gamma}}$ ). The interval $C_{\gamma \bar{\gamma}}>1.9$ corresponds to a Higgs BR into $\gamma \bar{\gamma}$ that is more than 3 times the $\operatorname{SM~} \mathrm{BR}(H \rightarrow \gamma \gamma)$, while $C_{Z \bar{\gamma}}>2.7$ corresponds to a Higgs BR into $Z \bar{\gamma}$ that is more than 9 times the $\operatorname{SM~} \mathrm{BR}(H \rightarrow Z \gamma)$.

The corresponding sensitivities on the $C_{\gamma \bar{\gamma}}$ and $C_{Z \bar{\gamma}}$ couplings at the ILC (foreseeing an initial $\sqrt{s}=250 \mathrm{GeV}$ phase, with a typical integrated luminosity of $250 \mathrm{fb}^{-1}$ ) can be 


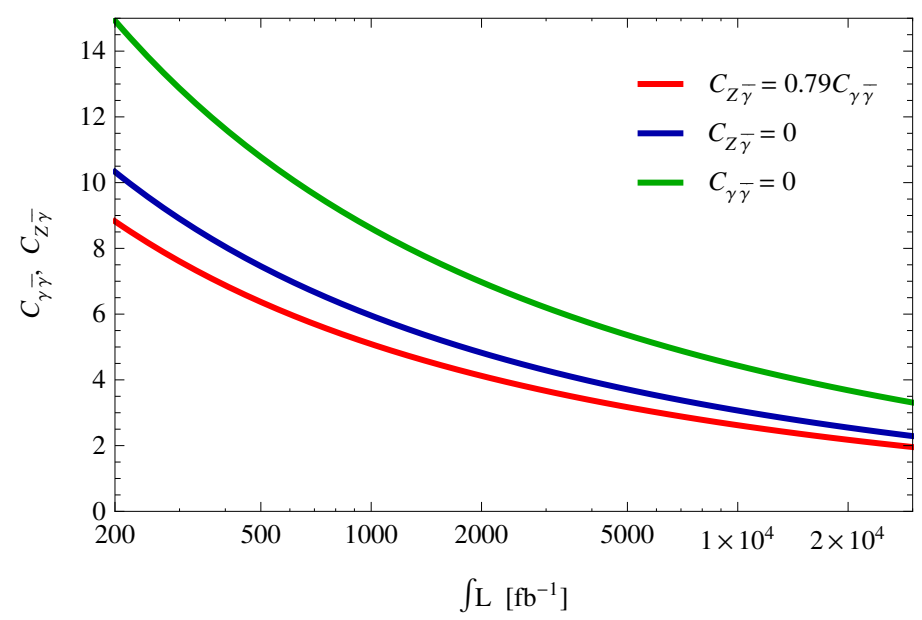

Figure 9. The $5 \sigma$-sensitivity bounds for $e^{+} e^{-} \rightarrow H \bar{\gamma}$ as a function of the integrated luminosity at $\sqrt{s}=240 \mathrm{GeV}$, when $C_{\gamma \bar{\gamma}}=0$ (green), $C_{Z \bar{\gamma}}=0$ (blue), and $C_{Z \bar{\gamma}}=0.79 C_{\gamma \bar{\gamma}}$ [with $C_{\gamma \bar{\gamma}}$ shown on the vertical axis] (red).

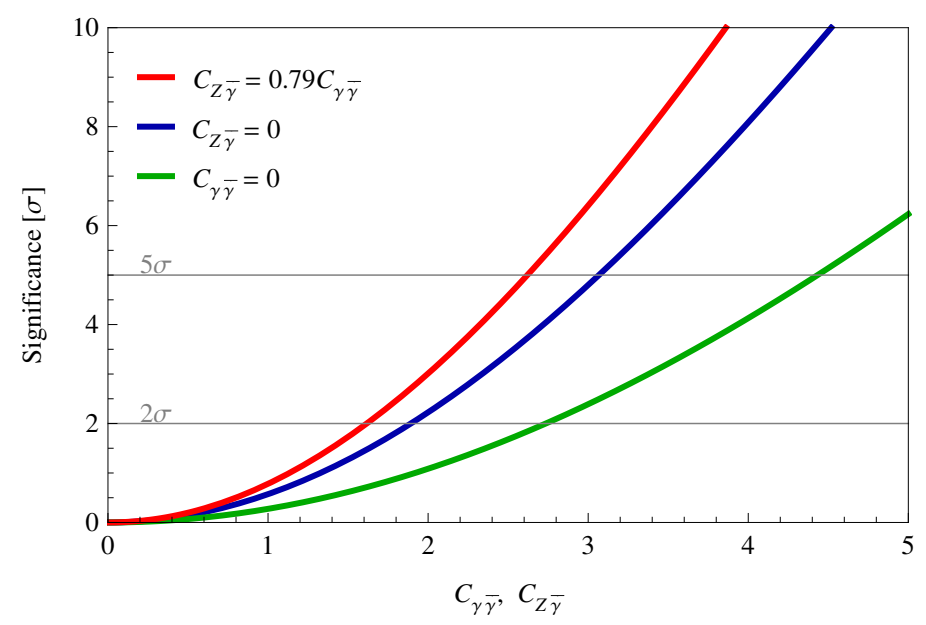

Figure 10. Signal significance $S / \sqrt{S+B}$ for $e^{+} e^{-} \rightarrow H \bar{\gamma}$ as a function of the couplings $C_{\gamma \bar{\gamma}}, C_{Z \bar{\gamma}}$, for $C_{\gamma \bar{\gamma}}=0$ (green), $C_{Z \bar{\gamma}}=0$ (blue), and $C_{Z \bar{\gamma}}=0.79 C_{\gamma \bar{\gamma}}$ [with $C_{\gamma \bar{\gamma}}$ shown on the horizontal axis] (red), for an integrated luminosity of $10 \mathrm{ab}^{-1}$, at $\sqrt{s}=240 \mathrm{GeV}$. The horizontal gray lines show the $5 \sigma$-discovery bound, and the $2 \sigma$ ( $\simeq 95 \%$ C.L. exclusion) level.

estimated from figure 9, and are about a factor 3 lower than the FCC-ee ones. The latter match sensitivities on the corresponding $\mathrm{BR}(H \rightarrow \gamma \bar{\gamma})$ and $\mathrm{BR}(H \rightarrow Z \bar{\gamma})$ that are smaller than the FCC-ee ones by an order of magnitude.

\section{A model of Flavor with dark photons}

In this section, we review the main aspects of the model proposed in [12], that provides a theoretical framework for the effective description given by the Lagrangian in eq. (2.1). Correspondingly, we will obtain predictions for the Higgs BR's for $H \rightarrow \gamma \bar{\gamma}$ and $H \rightarrow Z \bar{\gamma}$ (and relevant effective couplings) in terms of the fundamental parameters of the model. 


\subsection{The Lagragian}

The basic assumptions of the model introduced in [12] are the following. For each SM fermion there exists a dark fermion with same Flavor in a hidden sector, that is an heavier fermion replica which is a singlet under the SM gauge group. The chiral- and Flavorsymmetry spontaneus breaking is realized in the dark sector as described in the following, and communicated to the SM fermions via renormalizable and Flavor universal interactions mediated by messenger fields. The Yukawa couplings are radiatively generated, preserving approximately the same Flavor hierarchy structure of the dark-fermion sector. A similar approach has been proposed in [13-15], although in this case the dynamics responsible of the dark-fermion spectrum has not been discussed.

A simple choice for the messenger sector consists of a set of scalar messenger fields that, due to gauge invariance, have to be charged under the SM gauge group with the same quantum numbers as quarks and leptons. The relevant Lagrangian is [12]

$$
\mathcal{L}=\mathcal{L}_{\mathrm{SM}}^{Y=0}+\mathcal{L}_{\text {mes }}+\mathcal{L}_{\mathrm{DS}}
$$

where $\mathcal{L}_{\mathrm{SM}}^{Y=0}$ stands for the SM Lagrangian without tree-level Higgs Yukawa couplings, $\mathcal{L}_{\text {mes }}$ is the Lagrangian containing the messenger sector with its couplings to the SM and dark fields, and $\mathcal{L}_{\mathrm{DS}}$ is the dark-sector Lagrangian including the dynamics responsible of the Flavor hierarchy.

Dark fermions acquire an exponentially-spread mass spectrum from non-perturbative dynamical effects. In fact, the Lagrangian $\mathcal{L}_{\text {DS }}$, containing dark fermions and a dark photon, is given by [12],

$$
\mathcal{L}_{\mathrm{DS}}=i \sum_{i}\left(\bar{Q}^{U_{i}} \mathcal{D}_{\mu} \gamma^{\mu} Q^{U_{i}}+\bar{Q}^{D_{i}} \mathcal{D}_{\mu} \gamma^{\mu} Q^{D_{i}}\right)-\frac{1}{4} F_{\mu \nu} F^{\mu \nu}+\frac{1}{2 \Lambda^{2}} \partial^{\mu} F_{\mu \alpha} \partial_{\nu} F^{\nu \alpha}
$$

where $Q^{U_{i}}, Q^{D_{i}}$ are the dark fermion fields, partners in the hidden sector of the up $\left(U_{i}\right)$ and down $\left(D_{i}\right)$ quarks, $\mathcal{D}_{\mu}=\partial_{\mu}+i g \hat{Q} \bar{A}_{\mu}$ is the covariant derivative related to the $\mathrm{U}(1)_{F}$ gauge field $\bar{A}_{\mu}$ which is associated to the dark-photon, with $F_{\mu \alpha}$ the corresponding field strength, and $\hat{Q}$ the charge operator acting on the dark fermion fields. $\mathcal{L}_{\mathrm{DS}}$ can be extended to include also the SM leptonic sector in a straightforward way.

The last term in $\mathcal{L}_{\mathrm{DS}}$, involving only the $\mathrm{U}(1)_{F}$ gauge sector, corresponds to the socalled Lee-Wick term. It is a higher-derivative term, and the $\Lambda$ scale can be interpreted as the mass of the associated massive ghost. ${ }^{1}$ This term can trigger ChSB, and generate a mass spectrum non-perturbatively [51], by means of the Nambu-Jona-Lasinio mechanism [52, 53]. The following Dirac-fermion mass spectrum can be induced on the true vacuum [51]

$$
M_{Q_{i}}=\Lambda \exp \left\{-\frac{2 \pi}{3 \bar{\alpha}(\Lambda) q_{i}^{2}}+\frac{1}{4}\right\}
$$

\footnotetext{
${ }^{1}$ According to the Lee-Wick argument [54-56], the presence of a massive ghost field in the spectrum does not spoil unitarity, provided the massive ghost has a finite decay width, which is automatically satisfied in the present scenario.
} 
where $q_{i}$ is the $\mathrm{U}(1)_{F}$ charge of the Dirac fermion, and $\bar{\alpha}(\Lambda)$ the corresponding fine structure constant at the $\Lambda$ energy scale. This solution is manifestely non-perturbative as can be seen from the $\bar{\alpha}$ dependence in $M_{Q_{i}}$.

The Lagrangian $\mathcal{L}_{\text {mes }}$ in eq. (4.1) contains the messenger scalar fields,

$$
\mathcal{L}_{\text {mes }}=\mathcal{L}_{\text {mes }}^{0}+\mathcal{L}_{\text {mes }}^{\mathrm{I}},
$$

where $\mathcal{L}_{\text {mes }}^{0}$ is the kinetic Lagrangian for the messenger fields interacting with the SM gauge bosons, while $\mathcal{L}_{\text {mes }}^{\mathrm{I}}$ contains the messenger interactions with the dark fermions and the Higgs boson, which give rise to the effective Yukawa couplings.

The SM quark quantum numbers set the minimal matter in the messenger sector, which is given by

- $2 N_{f}$ complex scalar $\mathrm{SU}(2)_{L}$ doublets: $\hat{S}_{L}^{U_{i}}$ and $\hat{S}_{L}^{D_{i}}$,

- $2 N_{f}$ complex scalar $\mathrm{SU}(2)_{L}$ singlets: $S_{R}^{U_{i}}$ and $S_{R}^{D_{i}}$,

- one real $\mathrm{SU}(2)_{L} \times \mathrm{U}(1)_{Y}$ singlet scalar: $S_{0}$,

where $\hat{S}_{L}^{U_{i}\left(D_{i}\right)}=\left(\begin{array}{c}c S_{L_{1}}^{U_{i}\left(D_{i}\right)} \\ S_{L_{2}}^{U_{i}\left(D_{i}\right)}\end{array}\right), N_{f}=3$, and $i(=1,2,3)$ stands for the Flavor index for three fermion generations. The $\hat{S}_{L}^{U_{i}, D_{i}}$ and $S_{R}^{U_{i}, D_{i}}$ fields carry the SM quark quantum numbers, and the labels $L, R$ corresponds to the chirality of the associated SM fermions. They couple to the EW gauge bosons and to the gluons, as do squarks in the minimal supersymmetric extensions of the SM. Note that a minimal Flavor violation would require this Lagrangian to be invariant under $\mathrm{SU}\left(N_{F}\right)$, where $N_{F}=2 N_{f}$ is the number of Flavors.

The messenger mass structure can be described by four free universal mass terms in both the $\hat{S}_{L, R}^{U_{i}}$ and $\hat{S}_{L, R}^{D_{i}}$ sectors. Note that an even more minimal hypothesis of a common scalar mass for the $L$ and $R$ scalar sectors is also phenomenologically acceptable.

The Lagrangian $\mathcal{L}_{\text {mes }}^{I}$ for the messenger interactions with quarks and SM Higgs boson is

$$
\begin{aligned}
\mathcal{L}_{\text {mes }}^{I}= & g_{L}\left(\sum_{i=1}^{N_{f}}\left[\bar{q}_{L}^{i} Q_{R}^{U_{i}}\right] \hat{S}_{L}^{U_{i}}+\sum_{i=1}^{N_{f}}\left[\bar{q}_{L}^{i} Q_{R}^{D_{i}}\right] \hat{S}_{L}^{D_{i}}\right)+ \\
& +g_{R}\left(\sum_{i=1}^{N_{f}}\left[\bar{U}_{R}^{i} Q_{L}^{U_{i}}\right] S_{R}^{U_{i}}+\sum_{i=1}^{N_{f}}\left[\bar{D}_{R}^{i} Q_{L}^{D_{i}}\right] S_{R}^{D_{i}}\right)+ \\
& +\lambda_{S} S_{0}\left(\tilde{H}^{\dagger} S_{L}^{U_{i}} S_{R}^{U_{i} \dagger}+H^{\dagger} S_{L}^{D_{i}} S_{R}^{D_{i} \dagger}\right)+\text { h.c. }+V\left(S_{0}\right),
\end{aligned}
$$

where $S_{0}$ is a real singlet scalar, $V\left(S_{0}\right)$ its potential, $q_{L}^{i}, U_{R}^{i}, D_{R}^{i}$, stand for the SM fermions, and $H$ is the SM Higgs doublet, with $\tilde{H}=i \sigma_{2} H^{\star}$. Contractions on color indices are understood. The two constants $g_{L}$ and $g_{R}$ are Flavor-universal free parameters that are allowed to be in the perturbative region $g_{L, R}<1$.

The Lagrangian for the interaction of messenger scalars with the SM gauge bosons follows from the universal properties of gauge interactions. We stress that the messenger fields carry the same $\mathrm{U}(1)_{F}$ charges as the corresponding dark fermions. 


\begin{tabular}{|c||c|c|c|c|c|}
\hline Fields & Spin & $\mathrm{SU}(2)_{L}$ & $\mathrm{U}(1)_{Y}$ & $\mathrm{SU}(3)_{c}$ & $\mathrm{U}(1)_{F}$ \\
\hline$\hat{S}_{L}^{D_{i}}$ & 0 & $1 / 2$ & $1 / 3$ & 3 & $-q_{D_{i}}$ \\
\hline$\hat{S}_{L}^{U_{i}}$ & 0 & $1 / 2$ & $1 / 3$ & 3 & $-q_{U_{i}}$ \\
\hline$S_{R}^{D_{i}}$ & 0 & 0 & $-2 / 3$ & 3 & $-q_{D_{i}}$ \\
\hline$S_{R}^{U_{i}}$ & 0 & 0 & $4 / 3$ & 3 & $-q_{U_{i}}$ \\
\hline$Q^{D_{i}}$ & $1 / 2$ & 0 & 0 & 0 & $q_{D_{i}}$ \\
\hline$Q^{U_{i}}$ & $1 / 2$ & 0 & 0 & 0 & $q_{U_{i}}$ \\
\hline$S_{0}$ & 0 & 0 & 0 & 0 & 0 \\
\hline
\end{tabular}

Table 2. Spin and gauge quantum numbers for the strongly-interacting messenger fields and for dark fermions. $\mathrm{U}(1)_{F}$ is the gauge symmetry in the dark sector.

The messenger and dark-fermion quantum numbers are shown in table 2 (detailed in [12]). Finally, after the spontaneous symmetry breaking of the discrete $H \rightarrow-H$ parity symmetry that prevents tree-level Yukawa couplings, all Yukawa couplings are generated at one loop, and are finite at any order in perturbation theory [12-15]). Assuming almost degenerate diagonal messenger masses in the $\mathrm{L}$ and $\mathrm{R}$ sectors, one finds, from the one-loop vertex computation, that the effective Yukawa coupling associated to the quark $i$ is [12]

$$
Y^{i}=Y_{0}\left(x_{i}\right) \exp \left(-\frac{2 \pi}{3 \bar{\alpha}(\Lambda) q_{i}^{2}}\right)
$$

where the dark-fermion masses $M_{Q_{i}}$ have been replaced by eq. (4.3), and the one-loop function $Y_{0}\left(x_{i}\right)$ is given by

$$
Y_{0}\left(x_{i}\right)=\left(\frac{g_{L} g_{R}}{16 \pi^{2}}\right)\left(\frac{\mu_{S} \Lambda}{\bar{m}^{2}}\right) C_{0}\left(x_{i}\right),
$$

with $\mu_{S} \equiv \lambda_{S}\langle S\rangle$, and $x_{i}=M_{Q_{i}}^{2} / \bar{m}^{2}$. Also, $\bar{m}$ is the average mass of the messenger fields running in the loop, and $C_{0}(x)=(1-x(1-\log x)) /(1-x)^{2}{ }^{2}$

\section{2 $\operatorname{BR}(H \rightarrow \gamma \bar{\gamma})$ predictions}

The $C_{\gamma \gamma}, C_{\gamma \bar{\gamma}}$, and $C_{\bar{\gamma} \bar{\gamma}}$ coefficients entering the effective Lagrangian in eq. (2.1) have been computed in [35], as a function of the basic parameters of the model described in section 4.1.

In the $\bar{m}_{L}^{2} \simeq \bar{m}_{R}^{2}$ approximation of degenerate messenger masses in the left and righthanded sectors, corresponding to the mixing angle $\theta=\pi / 4$ (see [12] for notations), the Flavor-universal messenger mass matrix can be expressed in terms of two parameters, the average messenger mass $\bar{m}^{2}=\left(m_{L}^{2}+m_{R}^{2}\right) / 2$, and the mixing parameter $\xi \equiv \Delta^{2} / \bar{m}^{2}$. We then define the universal mixing parameters $\xi_{q}$ and $\xi_{l}$, corresponding to the messenger mixing parameters in the quark and lepton sectors, respectively. Note that, in the effective theory approximation, the Higgs and $Z$ masses can be both set to zero in loop functions, when terms $\mathcal{O}\left(m_{\mathrm{H}}^{2} / \bar{m}^{2}\right)$ are negligible.

\footnotetext{
${ }^{2}$ The above results hold for diagonal Yukawa couplings. They can be easily generalized to include the Cabibbo-Kobayashi-Maskawa (CKM) matrix entering the charged weak interactions as explained in [13, 14].
} 
Then, one finds ${ }^{3}$

$$
\begin{aligned}
C_{\gamma \bar{\gamma}} & =\sqrt{\frac{\bar{\alpha}}{\alpha}} \sum_{i=q, l} \frac{R_{1}^{i}}{12} \frac{\xi_{i}^{2}}{1-\xi_{i}^{2}}, \\
C_{\bar{\gamma} \bar{\gamma}} & =\frac{\bar{\alpha}}{\alpha} \sum_{i=q, l} \frac{R_{2}^{i}}{12} \frac{\xi_{i}^{2}}{1-\xi_{i}^{2}}, \\
C_{Z \bar{\gamma}} & =\sqrt{\frac{\bar{\alpha}}{\alpha}} \sum_{i=q, l} R_{Z \gamma}^{i} \frac{R_{1}^{i}}{12} \frac{\xi_{i}^{2}}{1-\xi_{i}^{2}}, \\
C_{\gamma \gamma} & =C_{\gamma \gamma}^{\mathrm{SM}}\left(1+\sum_{i=q, l} \frac{R_{0}^{i} \xi_{i}^{2}}{3 F\left(1-\xi_{i}^{2}\right)}\right) \\
C_{Z \gamma} & =C_{\gamma \gamma}^{\mathrm{SM}}\left(1+\sum_{i=q, l} R_{Z \gamma}^{i} \frac{R_{0}^{i} \xi_{i}^{2}}{3 F\left(1-\xi_{i}^{2}\right)}\right), \\
C_{g g} & =C_{g g}^{\mathrm{SM}}\left(1-\frac{\xi_{q}^{2}}{3 F_{q}\left(1-\xi_{q}^{2}\right)}\right),
\end{aligned}
$$

where $C_{\gamma \gamma}^{\mathrm{SM}}=\frac{1}{4} F, C_{g g}^{\mathrm{SM}}=F_{q}$, and the constants $R_{0,1,2}^{q, l}$ are given by

$$
\begin{array}{ll}
R_{0}^{q}=3 N_{c}\left(e_{U}^{2}+e_{D}^{2}\right), & R_{0}^{l}=3 e_{E}^{2}, \\
R_{1}^{q}=N_{c} \sum_{i=1}^{3}\left(e_{U} q_{U_{i}}+e_{D} q_{D_{i}}\right), & R_{1}^{l}=e_{E} \sum_{i=1}^{3}\left(q_{E_{i}}\right), \\
R_{2}^{q}=N_{c} \sum_{i=1}^{3}\left(q_{U_{i}}^{2}+q_{D_{i}}^{2}\right), & R_{2}^{l}=\sum_{i=1}^{3}\left(q_{E_{i}}^{2}+q_{\nu_{i}}^{2}\right),
\end{array}
$$

with $e_{U}=2 / 3, e_{D}=-1 / 3$, and $e_{E}=-1$, the electric charges for up-, down-quarks, and charged leptons, respectively. $F$ and $F_{q}$ are the usual SM loop factor given by

$$
F=F_{W}\left(\beta_{W}\right)+F_{F}, \quad F_{F}=\sum_{f} N_{c} Q_{f}^{2} F_{f}\left(\beta_{f}\right), \quad F_{q}=\sum_{f} F_{f}\left(\beta_{f}\right),
$$

with $N_{c}=(3) 1$ for leptons (quarks) respectively, $\beta_{W}=4 m_{W}^{2} / m_{\mathrm{H}}^{2}, \beta_{f}=4 m_{f}^{2} / m_{\mathrm{H}}^{2}$, and

$$
F_{W}(x)=2+3 x+3 x(2-x) f(x), \quad F_{f}(x)=-2 x(1+(1-x) f(x))
$$

where $f(x)=\arcsin ^{2}\left[\frac{1}{\sqrt{x}}\right]$, for $x \geq 1$, and $f(x)=-\frac{1}{4}\left(\log \left(\frac{1+\sqrt{1-x}}{1-\sqrt{1-x}}\right)-i \pi\right)^{2}$, for $x<1$. Including only the $W$ and top-quark loops in $F$, we get $F \simeq 6.5, F_{q} \simeq 1.8$ for $m_{\mathrm{H}}=$

${ }^{3}$ Note that, due to the Bose statistics of the messenger fields, the relative sign with respect to the SM contribution in the $H \rightarrow g g$ amplitude is predicted to be negative. Analogously, there is a negative relative sign with respect to the SM fermion contribution to the $H \rightarrow \gamma \gamma$ and $H \rightarrow \gamma Z$ amplitudes. 
$125 \mathrm{GeV}$. We will elaborate on the $R_{Z \gamma}^{q, l}$ constants in eq. (4.8) (parametrizing the ratio of the messenger couplings to the $Z$ and $\gamma$ ) at the end of this section. ${ }^{4}$

One can see that in the Higgs couplings in eq. (4.8), there is a clear non-decoupling effect, since the $C_{i j}$ coefficients do not vanish when $\left(\bar{m}^{2}, \Delta\right) \rightarrow \infty$, provided the ratio $\Delta / \bar{m}^{2}$ is finite.

The ratios $r_{i j}(i, j=\gamma, \bar{\gamma})$, defined in eq. (2.5), entering the model-independent BR's parametrization in eq. (2.6), and $r_{g g}$, defined in eq. (2.7), are then given by

$$
\begin{array}{rlrl}
r_{\gamma \bar{\gamma}} & =2\left(\sum_{i=l, q} X_{i} R_{1}^{i}\right)^{2}\left(\frac{\bar{\alpha}}{\alpha}\right), & r_{\bar{\gamma} \bar{\gamma}}=\left(\sum_{i=l, q} X_{i} R_{2}^{i}\right)^{2}\left(\frac{\bar{\alpha}}{\alpha}\right)^{2}, \\
r_{\gamma \gamma}=\left(\sum_{i=l, q} X_{i} R_{0}^{i}\right)^{2}, & r_{g g}=\frac{X_{q}^{2} F^{2}}{F_{q}^{2}},
\end{array}
$$

where the extra factor 2 in $r_{\gamma \bar{\gamma}}$ comes from statistics and

$$
X_{l(q)} \equiv \frac{\xi_{l(q)}^{2}}{3 F\left(1-\xi_{l(q)}^{2}\right)},
$$

with $R_{0,1,2}^{q, l}$ defined in eqs. (4.9).

The strength of the exotic contribution to $H \rightarrow \gamma \gamma$ is directly controlled by two mixing parameters, $\xi_{q}$ and $\xi_{l}$. On the other hand, the $H \rightarrow g g$ depends only on $\xi_{q}$, and can be constrained at the LHC by measuring Higgs production rates.

It is useful to connect the messenger-loop impact on the $H g g$ vertex expressed by $r_{g g}$ [as defined in eq. (2.7)] with the usual $k_{g}$ anomalous coupling of the $\mathrm{Hgg}$ interaction which enters the relation $C_{g g}=k_{g} C_{g g}^{\mathrm{SM}}$. By eq. (4.8), it is straightforward to see that $k_{g} \simeq 1-\sqrt{r_{g g}}$. Present data constraints $k_{g}$ at $68 \%$ of C.L. to be in the ranges $k_{g}=1.00_{-0.16}^{+0.23}$ (ATLAS collaboration [7]), and $k_{g}=0.76_{-0.13}^{+0.15}$ (CMS collaboration [8]). In the following, we assume $r_{g g}$ to be in the range $0 \lesssim r_{g g} \lesssim 0.4$.

In figure 11, we show $\mathrm{BR}(H \rightarrow \gamma \bar{\gamma})$ as a function of the $\mathrm{U}(1)_{F}$ fine structure constant evaluated at the average messenger mass, $\bar{\alpha}(\bar{m})$, for a few values of the ratios $r_{g g}$ and $r_{\gamma \gamma}$. We assume the charge normalization $q_{U_{3}}=1$ and $q_{L_{3}}=1$. The dashed blue lines correspond to the condition

$$
\frac{1}{2} \mathrm{BR}_{\gamma \gamma}^{\mathrm{SM}}<\mathrm{BR}_{\gamma \gamma}<2 \mathrm{BR}_{\gamma \gamma}^{\mathrm{SM}}
$$

with $\mathrm{BR}_{\gamma \gamma}^{\mathrm{SM}}=2.28 \times 10^{-3}$. The red dots correspond to fixed $\mathrm{BR}_{\bar{\gamma} \bar{\gamma}} \simeq \mathrm{BR}_{\text {inv }}$ values, where $\mathrm{BR}_{\text {inv }}$ is the Higgs invisible-decay $\mathrm{BR}$. Note that when colored messengers contribute to the $H \gamma \bar{\gamma}$ effective coupling, $\mathrm{BR}_{\gamma \bar{\gamma}}$ depends also on the sign of the $\mathrm{U}(1)_{F}$ charges, which are free parameters. Correspondingly, in figure 11, we show cases in which the $\mathrm{U}(1)_{F}$ charge sign in the quark sector gives either destructive or constructive interferences with

\footnotetext{
${ }^{4}$ We will neglect the $\bar{\alpha}$ running from the $\bar{m}$ scale to the characteristic low-energy scale entering the dark-photon vertex in $H \rightarrow \gamma \bar{\gamma}, \bar{\gamma} \bar{\gamma}, Z \bar{\gamma}$.
} 

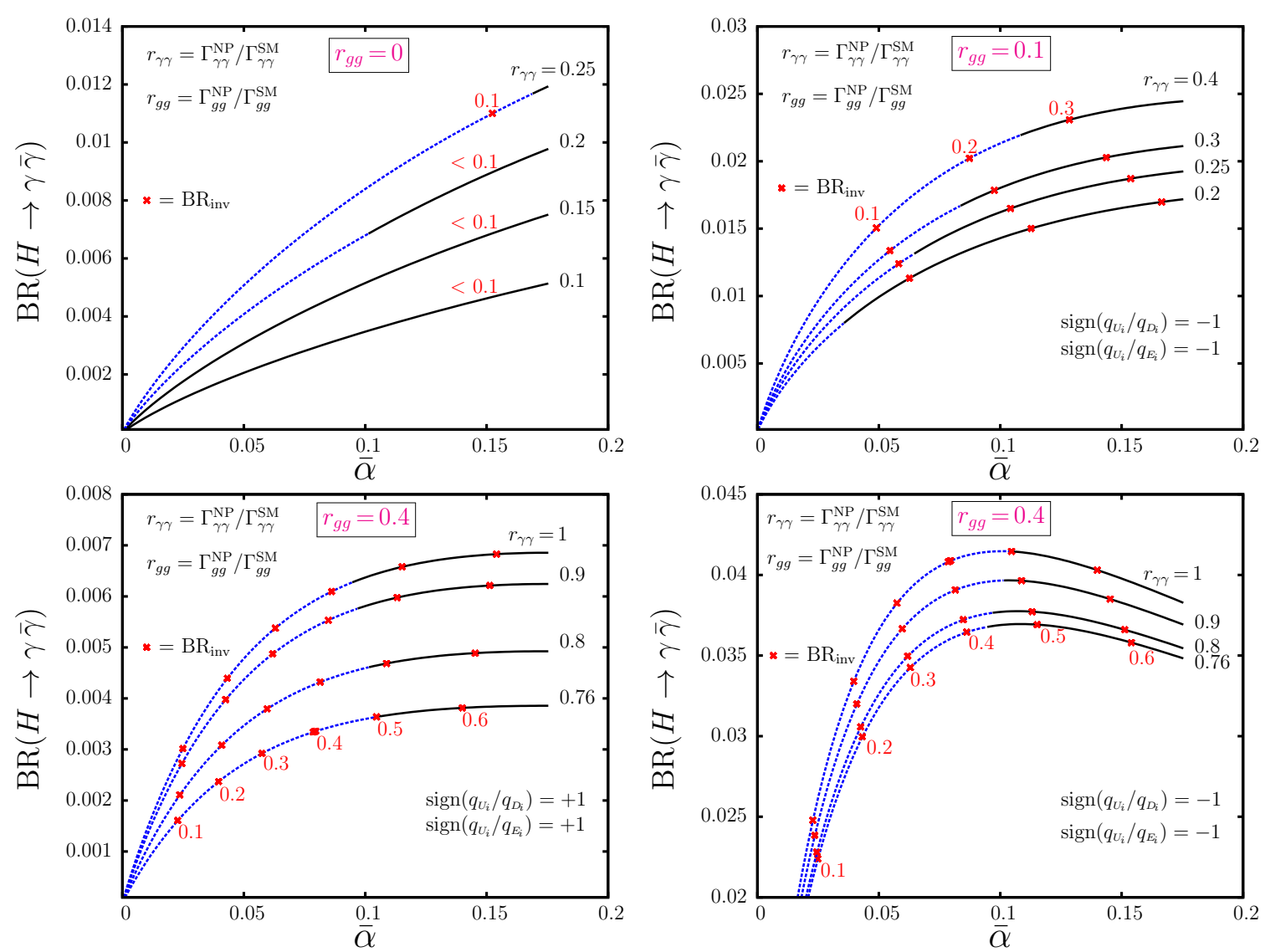

Figure 11. Predictions for $\operatorname{BR}(H \rightarrow \gamma \bar{\gamma})$ as functions of the $\mathrm{U}(1)_{F}$ fine structure constant $\bar{\alpha}$, for different values of $\mathrm{BR}_{\text {inv }}, r_{\gamma \gamma}$ and $r_{g g}$ in the full model; $r_{\gamma \gamma}$ satisfies the constraint $r_{\gamma \gamma} \gtrsim 1.9 r_{g g}$ (see text).

the electromagnetic charges. The maximum value of $\bar{\alpha} \simeq 0.18$ corresponds to $\bar{\alpha}(\Lambda) \simeq 1$ (see eq. (A.2) in the appendix for details).

In the upper-left plot in figure 11, we show the $\mathrm{BR}_{\gamma \bar{\gamma}}$ predictions for $r_{g g}=0$. The allowed $\mathrm{BR}_{\gamma \bar{\gamma}}$ values are at most about $1 \%$, and one has $\mathrm{BR}_{\gamma \bar{\gamma}}>\mathrm{BR}_{\gamma \gamma}^{\mathrm{SM}}$ only for $r_{\gamma \gamma} \gtrsim 0.1$, corresponding to a quite large mixing parameter $\left(\xi_{l}>0.82\right)$ in the leptonic messenger sector. The upper-right plot shows the case $r_{g g} \simeq 0.1$, assuming constructive $\mathrm{U}(1)_{F}$ charge interferences, corresponding to $\operatorname{sign}\left(q_{U_{i}} / q_{D_{i}}\right)=\operatorname{sign}\left(q_{U_{i}} / q_{E_{i}}\right)=-1$. In this plot, the minimum $r_{\gamma \gamma}$ value $\sim 0.2$ comes from the constraint $r_{\gamma \gamma} \gtrsim 1.9 r_{g g}$, arising from eqs. (4.13)-(4.14) as a result of the color and EW quantum numbers of the different amplitudes. $\mathrm{BR}_{\gamma \bar{\gamma}}$ above $2 \%$ are allowed in this case, corresponding to the range $\bar{\alpha} \sim 0.10-0.18$, with $r_{\gamma \gamma} \sim 0.4$.

The lower-left and lower-right plots match the largest allowed contribution from colored messengers $\left(r_{g g} \simeq 0.4\right.$, with $\left.r_{\gamma \gamma} \gtrsim 0.76\right)$, which corresponds to a mixing parameter $\xi_{q} \simeq 0.88$. The left and right plots correspond to the destructive and constructive effects of the $\mathrm{U}(1)_{F}$ charges, that is $\operatorname{sign}\left(q_{U_{i}} / q_{D_{i}}\right)=\operatorname{sign}\left(q_{U_{i}} / q_{E_{i}}\right)=1$, and $\operatorname{sign}\left(q_{U_{i}} / q_{D_{i}}\right)=$ $\operatorname{sign}\left(q_{U_{i}} / q_{E_{i}}\right)=-1$, respectively. For large colored messenger contributions, the actual $\mathrm{BR}_{\gamma \bar{\gamma}}$ value dramatically depends on the charge signs. In the case of constructive interferences, $\mathrm{BR}_{\gamma \bar{\gamma}}$ reaches the $3 \%-4 \%$ level, while in the destructive case it is always below $1 \%$. 
Summing up, large $\mathrm{BR}_{\gamma \bar{\gamma}}$ values up to $1 \%-4 \%$ are possible in this scenario. They correspond to the $\bar{\alpha}$ range expected from naturalness arguments applied to $\mathrm{U}(1)_{F}$ charges (see the appendix for more details). This $\mathrm{BR}_{\gamma \bar{\gamma}}$ range is equivalent to $C_{\gamma \bar{\gamma}}$ values up to about 5 in the Lagrangian in eq. (2.2), as shown in figure 4. As we have seen, in section 3, these $C_{\gamma \bar{\gamma}}$ values could be well inside the domain of sensitivity of the $e^{+} e^{-} \rightarrow H \bar{\gamma}$ searches at future $e^{+} e^{-}$colliders (cf. figures 9-10).

Before closing this section, we elaborate on the general connection between the $H Z \bar{\gamma}$ and $H \gamma \bar{\gamma}$ couplings, induced at one loop. This will depend on the spin and the SM gaugegroup representation of the particles running in the loop. On the other hand, here the $H Z \bar{\gamma}$ and $H \gamma \bar{\gamma}$ vertices are induced by scalar messenger fields in the $\mathrm{SU}(2)_{L} \times \mathrm{SU}(3)_{c}$ fundamental representation (cf. table 2), which gives a definite prediction for the $H Z \bar{\gamma}$ and $H \gamma \bar{\gamma}$ coupling ratio, and for the $R_{Z \gamma}^{q, l}$ terms entering the $C_{Z \bar{\gamma}}$ and $C_{Z \gamma}$ effective couplings in eq. (4.8).

Then we can now motivate the $R_{Z \gamma} \simeq 0.79$ scenario that we considered in the $e^{+} e^{-} \rightarrow$ $H \bar{\gamma}$ study of section 3. $R_{Z \gamma}$ can be defined (in absence of scalar mixing between the doublet and singlet scalars circulating in loops) by the relation

$$
C_{Z X}=R_{Z \gamma} C_{\gamma X}
$$

with $X=\gamma, \bar{\gamma}$, for the Higgs effective couplings in eqs. (2.2)-(2.3).

The $Z$-boson coupling to a scalar particle $i$ is given by

$$
R_{Z \gamma}^{i}=\frac{\left(1-\frac{Y^{i}}{Q^{i}}\right)-\sin ^{2} \theta_{W}}{\sin \theta_{W} \cos \theta_{W}}
$$

times the photon coupling to the same scalar, where $Y^{i}$ and $Q^{i}$ are the hypercharge and the electric charge of the scalar, and $\sin \theta_{W}$ is the Weinberg angle.

If the scalars in the loop are SM particle partners (as happens in SUSY or in the model in [12]), they will share the quantum numbers of the left- and right-handed SM fermions.

Then, for right-handed fermion partners $\left(Y^{R}=Q^{R}\right)$, and for left-handed electron partners $\left(Y^{\tilde{e}_{L}}=-\frac{1}{2}\right.$ and $\left.Q^{\tilde{e}_{L}}=-1\right)$, one has, respectively,

$$
R_{Z \gamma}^{R}=-\frac{\sin \theta_{W}}{\cos \theta_{W}} \simeq-0.55, \quad R_{Z \gamma}^{\tilde{e}_{L}}=\frac{\frac{1}{2}-\sin ^{2} \theta_{W}}{\sin \theta_{W} \cos \theta_{W}} \simeq 0.64
$$

The average $Z$-to- $\gamma$ coupling ratio for a pair of mass-degenerate right- and left-handed leptonic scalars is then

$$
R_{Z \gamma}^{\tilde{\ell}}=\frac{R_{Z \gamma}^{R}+R_{Z \gamma}^{\tilde{e}_{L}}}{2} \simeq 0.045
$$

For left-handed up- and down-type squarks $\left(Y^{\tilde{u}_{L}}=\frac{1}{4} Q^{\tilde{u}_{L}}\right.$ and $Y^{\tilde{d}_{L}}=-\frac{1}{2} Q^{\tilde{d}_{L}}$, respectively), one has instead

$$
R_{Z \gamma}^{\tilde{u}_{L}}=\frac{\frac{3}{4}-\sin ^{2} \theta_{W}}{\sin \theta_{W} \cos \theta_{W}} \simeq 1.2, \quad R_{Z \gamma}^{\tilde{d}_{L}}=\frac{\frac{3}{2}-\sin ^{2} \theta_{W}}{\sin \theta_{W} \cos \theta_{W}} \simeq 3.0 .
$$

The average contribution from a mass-degenerate pair of right- and left-handed up and down squarks is then $R_{Z \gamma}^{\tilde{u}}=\left(R_{Z \gamma}^{R}+R_{Z \gamma}^{\tilde{u}_{L}}\right) / 2 \simeq 0.34$, and $R_{Z \gamma}^{\tilde{d}}=\left(R_{Z \gamma}^{R}+R_{Z \gamma}^{\tilde{d}_{L}}\right) / 2 \simeq 1.23$, 
respectively. Assuming that also the up- and down-type scalars are mass degenerate, the net result from a squark doublet is then

$$
R_{Z \gamma}^{\tilde{q}}=\frac{R_{Z \gamma}^{\tilde{u}}+R_{Z \gamma}^{\tilde{d}}}{2} \simeq 0.79
$$

The same pattern for the $R_{Z \gamma}$ constants can be obtained in the model in [12], in the approximation of degenerate colored messenger scalars.

In section 3, we include the case $C_{Z \bar{\gamma}}=R_{Z \gamma}^{\tilde{q}} C_{\gamma \bar{\gamma}}=0.79 C_{\gamma \bar{\gamma}}$ among the benchmarks for the analysis of the $e^{+} e^{-} \rightarrow H \bar{\gamma}$ potential, corresponding to negligible leptonic contributions in the messenger loops.

\section{Conclusions}

Hidden sectors extending the SM theory can include an extra unbroken U(1) gauge symmetry. The corresponding gauge boson, a massless dark vector boson, can couple to the Higgs boson through renormalizable interactions involving scalar messengers, giving rise to effective $H \gamma \bar{\gamma}, H Z \bar{\gamma}$, and $H \bar{\gamma} \bar{\gamma}$ couplings. Since a massless dark photon is not revealed by collider detectors, the latter can be probed at the LHC and future colliders via the search for exotic Higgs decays into a photon or a $Z$ boson plus missing transverse energy, and the determination of the invisible Higgs decay width.

Another way to investigate the possible existence of the $H \gamma \bar{\gamma}$ and $H Z \bar{\gamma}$ couplings is the production at future $e^{+} e^{-}$colliders of a Higgs boson associated to a dark photon. The corresponding signature is very distinctive, since there is no irreducible SM background where the Higgs decay products are accompanied by a massless invisible system.

After introducing an effective Lagrangian description of the new Higgs interactions, we studied the potential of the $e^{+} e^{-} \rightarrow H \bar{\gamma}$ production for probing the corresponding $H \gamma \bar{\gamma}$ and $H Z \bar{\gamma}$ couplings, $C_{\gamma \bar{\gamma}}$ and $C_{Z \bar{\gamma}}$. A most useful strategy for enhancing the $S / B$ ratio turns out to be a selection on the small values of the invisible-system invariant mass. We found that, at $\sqrt{s}=240 \mathrm{GeV}$, with the integrated luminosity foreseen at the FCC-ee $(\sim 10$ $\mathrm{ab}^{-1}$ ), one can exclude at $95 \%$ C.L. the ranges $C_{\gamma \bar{\gamma}}>1.9$ (for $C_{Z \bar{\gamma}}=0$ ), $C_{Z \bar{\gamma}}>2.7$ (for $C_{\gamma \bar{\gamma}}=0$ ), and $C_{\gamma \bar{\gamma}}>1.6$ (for $C_{Z \bar{\gamma}}=0.79 C_{\gamma \bar{\gamma}}$ ). The interval $C_{\gamma \bar{\gamma}}>1.9$ corresponds to $\mathrm{BR}(H \rightarrow \gamma \bar{\gamma})>3 \mathrm{BR}_{\mathrm{SM}}(H \rightarrow \gamma \gamma)$, while, excluding $C_{Z \bar{\gamma}}>2.7$, one excludes $\mathrm{BR}(H \rightarrow$ $Z \bar{\gamma})>9 \mathrm{BR}_{\mathrm{SM}}(H \rightarrow Z \gamma)$. The corresponding BR bounds for the Higgs decay into a dark photon at the ILC with $\sqrt{s}=250 \mathrm{GeV}$, and $\sim 250 \mathrm{fb}^{-1}$ of integrated luminosity, are about an order of magnitude looser than the FCC-ee ones.

Of course, in order to fully assess the $e^{+} e^{-}$collision potential, one would need an estimate of the corresponding LHC sensitivity to the $H \gamma \bar{\gamma}$ and $H Z \bar{\gamma}$ couplings. This would in particular allow to figure out whether, in case of negative findings at the forthcoming LHC runs, there will indeed be some unexplored parameter space left that can be covered by searches in $e^{+} e^{-}$collisions. Studies to evaluate the LHC sensitivity to the $H \rightarrow \gamma \bar{\gamma}$ channel at Run 1 have just been started. A parton-level analysis leads to a bound on $\operatorname{BR}(H \rightarrow$ $\gamma \bar{\gamma})$ of about half a percent at $95 \%$ C.L. from the $8-\mathrm{TeV}$ data set [35], corresponding to the exclusion of the range $C_{\gamma \bar{\gamma}}>1.6$ (cf. figure 4). On the other hand, since most 
of the background to the $H \rightarrow \gamma \bar{\gamma}$ signal in $p p$ collisions comes from jet- and photonmismeasurement effects, a thorough detector simulation would be needed to make a robust sensitivity statement on the present data set at the LHC. Studies at $13-14 \mathrm{TeV}$ are expected to increase the sensitivity on a purely signal-statistics basis, but will be affected by harsher experimental conditions, that make the reconstruction of relatively small missing transverse energies quite critical. The extrapolation of present $8-\mathrm{TeV}$ results to larger $p p$ c.m. energies will then require an even more careful analysis. Similar considerations apply to the LHC searches for a $H \rightarrow Z \bar{\gamma}$ signal, where the expected missing transverse energy is even lower than in the $H \rightarrow \gamma \bar{\gamma}$ case.

Predictions for the $\mathrm{BR}(H \rightarrow \gamma \bar{\gamma})$ in the framework of the Flavor model proposed in [12] have also been presented. Due to non-decoupling effects, $\operatorname{BR}(H \rightarrow \gamma \bar{\gamma})$ turns out to be directly proportional to the mixing parameters $\xi_{q, l}$ in the scalar messenger sector, and to the $\mathrm{U}(1)_{F}$ coupling constant $\bar{\alpha}$, times some SM couplings. Remarkably, quite high $\xi_{q, l}$ mixings are required in order to generate the Yukawa couplings radiatively, and avoid large fine tuning in the Higgs sector [12]. At the same time, large (but still in the perturbative range) $\bar{\alpha}$ couplings are expected, in order to avoid fine-tuning among the dark fermion charges. As a consequence, in the scenario [12], $\mathrm{BR}(H \rightarrow \gamma \bar{\gamma})$ can be naturally large, and in the ballpark of sensitivity of the LHC and future colliders.

In conclusion, although realistic LHC sensitivity studies for the Higgs couplings to dark photons are still to come, one expects quite a lot of complementarity of the LHC and future $e^{+} e^{-}$capabilities to probe new exotic Higgs-boson interactions with dark photons, thanks to the extremely clean $e^{+} e^{-}$experimental conditions.

\section{Acknowledgments}

E.G. would like to thank the PH-TH division of CERN for its kind hospitality during the preparation of this work. This work was supported by the ESF grants MTT59, MTT60, by the recurrent financing project SF0690030s09, and by the European Union through the European Regional Development Fund.

\section{A Coupling strength in the dark sector}

One of the most interesting property of the Flavor model in [12] is that the value of the $\mathrm{U}(1)_{F}$ coupling strength in the dark sector, $\bar{\alpha}$, can be connected to the charge splitting among two dark-fermion generations in the lepton or quark sector. In the following, we present such a prediction.

A crucial assumption in the model is the one of minimal Flavor violation, implying that the only source of Flavor violation comes from the $\mathrm{U}(1)_{F}$ charges. We first define $\bar{\alpha}(\Lambda)$ by normalizing to 1 the largest $\mathrm{U}(1)_{F}$ charge, which is the one associated to the dark-fermion partner of the top quark. Then, according to eq. (4.6), we get

$$
1 / \bar{\alpha}(\Lambda) \simeq \frac{3}{2 \pi} \frac{q_{Q_{i}}^{2}}{1-q_{Q_{i}}^{2}} \log \frac{m_{\mathrm{t}}}{m_{i}},
$$


where $m_{\mathrm{t}}$ is the top-quark mass, $m_{i}$ stands for another generic quark mass, and $q_{Q_{i}}$ is the $\mathrm{U}(1)_{F}$ charge of the corresponding dark-fermion partner $Q_{i}{ }^{5}$ Then, by fixing the ratio of two $q_{Q_{i}}$ charges in eq. (A.1), one can predict $\bar{\alpha}(\Lambda)$, as well as all the remaining $\mathrm{U}(1)_{F}$ charges. For instance, by requiring a charge splitting of $10 \%$ among the $\mathrm{U}(1)_{F}$ charges of the dark-fermion partners of the third quark generation, namely $q_{U_{3}} \simeq 1$ and $q_{D_{3}} \simeq 0.9$, we get $\bar{\alpha}(\Lambda) \simeq 0.13$, and also $q_{U_{2}} \simeq 0.87, q_{D_{2}} \simeq 0.82$, and $q_{U_{1}} \simeq 0.78, q_{D_{1}} \simeq 0.76$, where for the different quark masses we assumed the central values in [57].

On the other hand, in order to obtain a weakly coupled $\mathrm{U}(1)_{F}$ theory, with $\bar{\alpha}(\Lambda) \ll$ $10^{-2}$, a charge splitting $\ll 1 \%$ is required, leading to an unnatural fine tuning among the $\mathrm{U}(1)_{F}$ charges. Remarkably, the same conclusion holds when extracting $\bar{\alpha}(\Lambda)$ from the eq. (A.1) applied to the purely EW dark sector. In this case, assuming $q_{E_{3}}=1$ and $q_{E_{2}}=0.9$ for the dark fermions associated to the $\tau$ and $\mu$ leptons, respectively, one obtains $\bar{\alpha}=0.17$, which is of the same order of the coupling strength for dark fermions in the quark sector. In order to avoid an unnatural fine-tuning among the $\mathrm{U}(1)_{F}$ charges, eq. (A.1) suggests a large, but maybe still perturbative, $\mathrm{U}(1)_{F}$ coupling in the dark sector.

Actually, in [12] the scale $\Lambda$ can be many orders of magnitude above the messenger mass scale, and it is useful to have the $\bar{\alpha}$ value at low energy (for instance, at the average messenger mass $\bar{m}$ ), as a function of the charge splitting of a pair of different dark fermions. Indeed, it is the low-energy $\bar{\alpha}$ that enters $\mathrm{BR}(H \rightarrow \gamma \bar{\gamma})$ and $\mathrm{BR}(H \rightarrow \bar{\gamma} \bar{\gamma})$. We then need first to connect $\bar{\alpha}(\Lambda)$ to $\bar{\alpha}(\bar{m})$ by solving the appropriate renormalization group equations for the $\mathrm{U}(1)_{F} \beta$-function. Due to the large energy gap between $\Lambda$ and $\bar{m}$, all dark-fermion and messenger masses can be approximated to a common low-energy scale around $\bar{m}$, neglecting the running between different mass thresholds at low energy. Then, including the dark-fermion and messenger contributions to the one-loop beta-function, as well as the dark-fermion mass definition in eq. (4.3), one can remove the explicit dependence on the $\Lambda$ scale, by the expression

$$
\bar{\alpha}(\Lambda)=\bar{\alpha}(\bar{m})\left(1+R_{2}^{q}\left(\frac{4+3 N_{c}^{2}}{9 N_{c}}\right)+R_{2}^{l} \frac{7}{9}\right)
$$

where $R_{2}^{q, l}$ are defined in eq. (4.9). The peculiar solution in eq. (A.2) arises from reabsorbing the usual $\log \left(\Lambda / M_{Q_{i}}\right)$ term into the dark-fermion mass definition in eq. (4.3).

In figure 12 , the $\bar{\alpha}$ behavior is shown as a function of the $\mathrm{U}(1)_{F}$ charge splitting $\delta_{3}$ associated to the third generation of quarks, with $q_{Q_{D_{3}}}=q_{Q_{U_{3}}}\left(1-\delta_{3}\right)$. We assume the charge normalization $q_{U_{3}}=1$, and also $q_{L_{3}}=1$ for the $\mathrm{U}(1)_{F}$ charges of the dark fermions and messengers of the third-generation leptonic sector. The end point at $\delta_{3}=0.39$ corresponds to $\bar{\alpha}(\Lambda)=1$.

By requiring a natural charge splitting that is not smaller than $20 \%, \bar{\alpha}$ turns out to be quite strong, $0.04<\bar{\alpha}<0.18$, but still in the perturbative regime. Then, one obtains quite naturally large values for the $H \rightarrow \gamma \bar{\gamma}$ branching ratio.

\footnotetext{
${ }^{5}$ We have used the property that the loop function $Y_{0}\left(x_{i}\right)$, as defined in eq. (4.7), has a weak dependence on the dark-fermion mass $M_{Q_{i}}$, and can be approximated to a constant.
} 


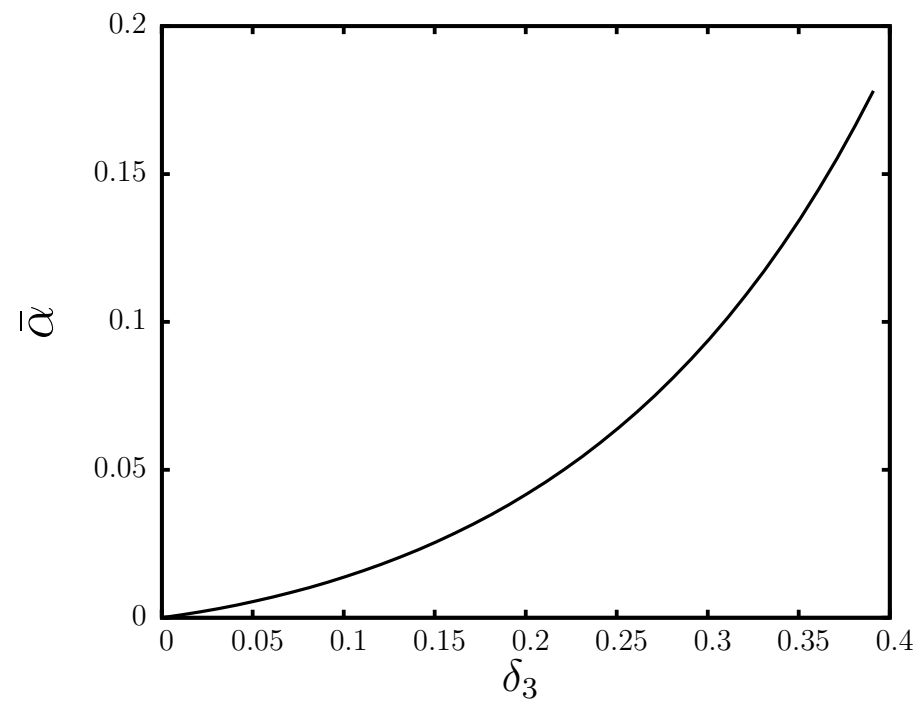

Figure 12. Predictions for $\bar{\alpha}$ at the low-energy messenger scale $\bar{m}$, as a function of the $\mathrm{U}(1)_{F}$ charge splitting in the third generation, defined by $q_{Q_{D_{3}}}=q_{Q_{U_{3}}}\left(1-\delta_{3}\right)$.

Open Access. This article is distributed under the terms of the Creative Commons Attribution License (CC-BY 4.0), which permits any use, distribution and reproduction in any medium, provided the original author(s) and source are credited.

\section{References}

[1] ATLAS collaboration, Observation of a new particle in the search for the Standard Model Higgs boson with the ATLAS detector at the LHC, Phys. Lett. B 716 (2012) 1 [arXiv: 1207.7214] [INSPIRE].

[2] CMS collaboration, Observation of a new boson at a mass of $125 \mathrm{GeV}$ with the CMS experiment at the LHC, Phys. Lett. B 716 (2012) 30 [arXiv:1207.7235] [INSPIRE].

[3] F. Englert and R. Brout, Broken Symmetry and the Mass of Gauge Vector Mesons, Phys. Rev. Lett. 13 (1964) 321 [inSPIRE].

[4] P.W. Higgs, Broken symmetries, massless particles and gauge fields, Phys. Lett. 12 (1964) 132 [INSPIRE].

[5] P.W. Higgs, Broken Symmetries and the Masses of Gauge Bosons, Phys. Rev. Lett. 13 (1964) 508 [INSPIRE].

[6] G.S. Guralnik, C.R. Hagen and T.W.B. Kibble, Global Conservation Laws and Massless Particles, Phys. Rev. Lett. 13 (1964) 585 [InSPIRE].

[7] ATLAS collaboration, Updated coupling measurements of the Higgs boson with the ATLAS detector using up to $25 \mathrm{fb}^{-1}$ of proton-proton collision data, ATLAS-CONF-2014-009 (2014) [ATLAS-COM-CONF-2014-013] [INSPIRE].

[8] CMS collaboration, Precise determination of the mass of the Higgs boson and studies of the compatibility of its couplings with the standard model, CMS-PAS-HIG-14-009 (2014) [INSPIRE]. 
[9] Planck collaboration, P.A.R. Ade et al., Planck 2013 results. XVI. Cosmological parameters, Astron. Astrophys. 571 (2014) A16 [arXiv: 1303.5076] [INSPIRE].

[10] Planck collaboration, P.A.R. Ade et al., Planck 2015 results. XIII. Cosmological parameters, arXiv: 1502.01589 [INSPIRE].

[11] B. Patt and F. Wilczek, Higgs-field portal into hidden sectors, hep-ph/0605188 [INSPIRE].

[12] E. Gabrielli and M. Raidal, Exponentially spread dynamical Yukawa couplings from nonperturbative chiral symmetry breaking in the dark sector, Phys. Rev. D 89 (2014) 015008 [arXiv: 1310.1090] [INSPIRE].

[13] E. Ma, The Higgs connection - Flavor and dark matter, Int. J. Mod. Phys. A 29 (2014) 1430034 [arXiv: 1402.3841] [INSPIRE].

[14] E. Ma, Radiative Origin of All Quark and Lepton Masses through Dark Matter with Flavor Symmetry, Phys. Rev. Lett. 112 (2014) 091801 [arXiv:1311.3213] [InSPIRE].

[15] S. Fraser and E. Ma, Anomalous Higgs Yukawa Couplings, Europhys. Lett. 108 (2014) 1002 [arXiv: 1402.6415] [INSPIRE].

[16] B. Holdom, Two U(1)'s and Epsilon Charge Shifts, Phys. Lett. B 166 (1986) 196 [INSPIRE].

[17] S.A. Abel, M.D. Goodsell, J. Jaeckel, V.V. Khoze and A. Ringwald, Kinetic Mixing of the Photon with Hidden U(1)s in String Phenomenology, JHEP 07 (2008) 124 [arXiv: 0803.1449] [INSPIRE].

[18] M. Goodsell, J. Jaeckel, J. Redondo and A. Ringwald, Naturally Light Hidden Photons in LARGE Volume String Compactifications, JHEP 11 (2009) 027 [arXiv:0909.0515] [INSPIRE].

[19] S. Baek, P. Ko and W.-I. Park, Singlet Portal Extensions of the Standard Seesaw Models to a Dark Sector with Local Dark Symmetry, JHEP 07 (2013) 013 [arXiv:1303.4280] [INSPIRE].

[20] S. Andreas, M.D. Goodsell and A. Ringwald, Hidden Photons in connection to Dark Matter, AIP Conf. Proc. 1563 (2013) 114 [arXiv: 1306.1168] [INSPIRE].

[21] H. An, M. Pospelov and J. Pradler, New Light on Dark Photons, arXiv:1309.6599 [INSPIRE].

[22] S. Baek, P. Ko and W.-I. Park, Hidden sector monopole, vector dark matter and dark radiation with Higgs portal, JCAP 10 (2014) 067 [arXiv:1311.1035] [INSPIRE].

[23] H. Vogel and J. Redondo, Dark Radiation constraints on minicharged particles in models with a hidden photon, JCAP 02 (2014) 029 [arXiv:1311.2600] [INSPIRE].

[24] K. Petraki, L. Pearce and A. Kusenko, Self-interacting asymmetric dark matter coupled to a light massive dark photon, JCAP 07 (2014) 039 [arXiv: 1403.1077] [INSPIRE].

[25] V.V. Khoze and G. Ro, Dark matter monopoles, vectors and photons, JHEP 10 (2014) 061 [arXiv: 1406.2291] [INSPIRE].

[26] J. Jaeckel, S. Roy and C.J. Wallace, Hidden photons with Kaluza-Klein towers, arXiv: 1408.0019 [INSPIRE].

[27] B. Brahmachari and A. Raychaudhuri, Kinetic mixing and symmetry breaking dependent interactions of the dark photon, Nucl. Phys. B 887 (2014) 441 [arXiv:1409.2082] [INSPIRE].

[28] P. Ko, Dark matter, dark radiation and Higgs phenomenology in the hidden sector DM models, arXiv: 1503.05412 [INSPIRE]. 
[29] D0 collaboration, V.M. Abazov et al., Search for dark photons from supersymmetric hidden valleys, Phys. Rev. Lett. 103 (2009) 081802 [arXiv:0905.1478] [InSPIRE].

[30] S. Andreas, C. Niebuhr and A. Ringwald, New Limits on Hidden Photons from Past Electron Beam Dumps, Phys. Rev. D 86 (2012) 095019 [arXiv:1209.6083] [InSPIRE].

[31] HADES collaboration, G. Agakishiev et al., Searching a Dark Photon with HADES, Phys. Lett. B 731 (2014) 265 [arXiv:1311.0216] [INSPIRE].

[32] BABAR collaboration, J.P. Lees et al., Search for a Dark Photon in $e^{+} e^{-}$Collisions at BaBar, Phys. Rev. Lett. 113 (2014) 201801 [arXiv:1406.2980] [INSPIRE].

[33] D. Curtin, R. Essig, S. Gori and J. Shelton, Illuminating Dark Photons with High-Energy Colliders, JHEP 02 (2015) 157 [arXiv: 1412.0018] [INSPIRE].

[34] Belle collaboration, I. Jaegle, Search for the dark photon and the dark Higgs boson at Belle, arXiv: 1502.00084 [INSPIRE].

[35] E. Gabrielli, M. Heikinheimo, B. Mele and M. Raidal, Dark photons and resonant monophoton signatures in Higgs boson decays at the LHC, Phys. Rev. D 90 (2014) 055032 [arXiv:1405.5196] [INSPIRE].

[36] T. Behnke et al., The International Linear Collider Technical Design Report - Volume 1: Executive Summary, arXiv:1306.6327 [INSPIRE].

[37] M. Aicheler et al., A Multi-TeV Linear Collider Based on CLIC Technology: CLIC Conceptual Design Report, CERN-2012-007 (2012) [SLAC-R-985] [KEK-Report-2012-1] [PSI-12-01] [JAI-2012-001] [INSPIRE].

[38] Future Circular Collider study, https://espace2013.cern.ch/fcc/Pages/default.aspx, (2015).

[39] TLeP Design Study Working Group, M. Bicer et al., First Look at the Physics Case of TLEP, JHEP 01 (2014) 164 [arXiv:1308.6176] [INSPIRE].

[40] A. Barroso, J. Pulido and J.C. Romao, Higgs production at $e^{+} e^{-}$colliders, Nucl. Phys. B 267 (1986) 509 [INSPIRE].

[41] A. Abbasabadi, D. Bowser-Chao, D.A. Dicus and W.W. Repko, Higgs photon associated production at eè colliders, Phys. Rev. D 52 (1995) 3919 [hep-ph/9507463] [INSPIRE].

[42] A. Djouadi, V. Driesen, W. Hollik and J. Rosiek, Associated production of Higgs bosons and a photon in high-energy $e^{+} e^{-}$collisions, Nucl. Phys. B 491 (1997) 68 [hep-ph/9609420] [INSPIRE].

[43] Q.-H. Cao, H.-R. Wang and Y. Zhang, Probing $H Z \gamma$ and $H \gamma \gamma$ anomalous couplings in the process of $e^{+} e^{-} \rightarrow H \gamma$, arXiv: 1503.05060 [INSPIRE].

[44] H. Baer et al., The International Linear Collider Technical Design Report - Volume 2: Physics, arXiv:1306.6352 [INSPIRE].

[45] D.M. Asner et al., ILC Higgs White Paper, arXiv:1310.0763 [INSPIRE].

[46] S. Dawson et al., Working Group Report: Higgs Boson, arXiv:1310.8361 [InSPIRE].

[47] A. Alloul, N.D. Christensen, C. Degrande, C. Duhr and B. Fuks, FeynRules $2.0-A$ complete toolbox for tree-level phenomenology, Comput. Phys. Commun. 185 (2014) 2250 [arXiv: 1310.1921] [INSPIRE].

[48] J. Alwall et al., The automated computation of tree-level and next-to-leading order differential cross sections and their matching to parton shower simulations, JHEP 07 (2014) 079 [arXiv: 1405.0301] [INSPIRE]. 
[49] T. Behnke et al., The International Linear Collider Technical Design Report - Volume 4: Detectors, arXiv:1306.6329 [INSPIRE].

[50] K. Seidel, F. Simon, M. Tesar and S. Poss, Top quark mass measurements at and above threshold at CLIC, Eur. Phys. J. C 73 (2013) 2530 [arXiv:1303.3758] [InSPIRE].

[51] E. Gabrielli, On the dynamical breaking of chiral symmetry: A New mechanism, Phys. Rev. D 77 (2008) 055020 [arXiv: 0712.2208] [INSPIRE].

[52] Y. Nambu and G. Jona-Lasinio, Dynamical Model of Elementary Particles Based on an Analogy with Superconductivity. 1., Phys. Rev. 122 (1961) 345 [INSPIRE].

[53] Y. Nambu and G. Jona-Lasinio, Dynamical Model Of Elementary Particles Based On An Analogy With Superconductivity. II, Phys. Rev. 124 (1961) 246 [INSPIRE].

[54] T.D. Lee and G.C. Wick, Questions of Lorentz Invariance in Field Theories With Indefinite Metric, Phys. Rev. D 3 (1971) 1046 [inSPIRE].

[55] T.D. Lee and G.C. Wick, Finite Theory of Quantum Electrodynamics, Phys. Rev. D 2 (1970) 1033 [INSPIRE].

[56] R.E. Cutkosky, P.V. Landshoff, D.I. Olive and J.C. Polkinghorne, A non-analytic $S$ matrix, Nucl. Phys. B 12 (1969) 281 [inSPIRE].

[57] Particle Data Group collaboration, K. Olive et al., Review of Particle Physics, Chin. Phys. C 38 (2014) 090001 [InSPIRE]. 